\title{
Reactive or transgenic increase in microglial TYROBP reveals a TREM2-independent TYROBP-APOE link in wild-type and Alzheimer's-related mice
}

\begin{abstract}
Mickael Audrain ${ }^{1}$, Jean-Vianney Haure-Mirande ${ }^{1}$, Justyna Mleczkoํ․ Minghui Wang², Jennifer K. Griffin ${ }^{3}$, Paul Fraser ${ }^{3}$, Bin Zhang ${ }^{2}$, Sam Gandy ${ }^{1,4,5,{ }^{*}}$ and Michelle E. Ehrlich ${ }^{1, *}$
\end{abstract}

${ }^{1}$ Department of Neurology, Icahn School of Medicine at Mount Sinai, New York, NY 10029, USA

${ }^{2}$ Department of Genetics and Genomic Sciences and Icahn Institute of Genomic Sciences, Icahn School of Medicine at Mount Sinai, New York, NY 10029, USA

${ }^{3}$ Tanz Centre for Research in Neurodegenerative Diseases, University of Toronto, Toronto, ON. M5T OS8, Canada

${ }^{4}$ National Institute on Aging-Designated Alzheimer's Disease Research Center and Department of Psychiatry, Icahn School of Medicine at Mount Sinai, New York, NY 10029, USA

${ }^{5}$ Research and Development, James J. Peters Veterans Affairs Medical Center, Bronx, NY 10468, USA

*Correspondence: samuel.gandy@mssm.edu; michelle.ehrlich@mssm.edu 


\section{ABSTRACT}

Microglial TYROBP (also known as DAP12) has been identified by computational transcriptomics as a network hub and driver in late-onset sporadic Alzheimer's disease (AD) and as an important regulator of the microglial environmental sensing function. TYROBP is the transmembrane adaptor of AD-related receptors TREM2 and CR3, but importantly, TYROBP interacts with many other receptors, and little is known about its roles in microglial action and/or in the pathogenesis of AD. Herein, using dual RNA in situ hybridization and immunohistochemistry, we demonstrate that endogenous Tyrobp transcription is increased specifically in recruited microglia in the brains of wild-type and AD-related mouse models. To determine whether chronically elevated TYROBP might modify microglial phenotype and/or progression of $\mathrm{AD}$ pathogenesis, we generated a novel transgenic mouse overexpressing TYROBP in microglia. TYROBP-overexpressing mice were crossed with either $A P P / P S E N 1$ or $M A P T^{P 301 S}$ mice, resulting in a decrease of the amyloid burden in the former and an increase of TAU phosphorylation in the latter. Apolipoprotein E (Apoe) transcription was upregulated in $M A P T^{P 301 S}$ mice overexpressing TYROBP and transcription of genes previously associated with Apoe, including $A x l, C c l 2, \operatorname{Tg} f \beta$ and Il6, was altered in both APP/PSEN1 and MAPT ${ }^{P 301 S}$ mice overexpressing TYROBP. Lastly, Tyrobp and Apoe mRNAs were clearly increased in Trem2-null mice in microglia recruited around a cortical stab injury or amyloid- $\beta$ (A $\beta)$ deposits. Conversely, microglial Apoe transcription was dramatically diminished when Tyrobp was absent. Our results provide compelling evidence that TYROBP-APOE signaling in the microglial sensome does not require TREM2. We propose that activation of a TREM2independent TYROBP-APOE signaling could be an early or even initiating step in the transformation of microglia from the homeostatic phenotype to the Disease-Associated Microglia (DAM) phenotype.

KEYWORDS: Tyrobp, Dap12, Apoe, Trem2, microglia, Alzheimer's disease, amyloid, APP/PSEN1, tauopathy, PS19, RNAscope, DAM 


\section{INTRODUCTION}

Microglia play a sentinel role in the brain, capable of detecting a wide variety of environmental stimuli, including microbial pathogens, aggregated proteins (such as amyloid- $\beta$; A $\beta$ ) and cellular debris (such as membrane fragments). This sensing activity is an essential part of the host response and is broad in its scope, sometimes triggering homeostatic adjustment, while, at other times, host defense. Microglia are also of interest in neurodegenerative diseases due to proteinopathies, e.g. Alzheimer's disease (AD), in which large genetic studies have reported increased disease risk linked to many loci associated with microglial genes implicated in clearance of A $\beta$ peptides ${ }^{1-7}$. More recently, transcriptomic analyses have revealed distinct profiles and signatures for microglia associated with aging and age-related diseases, indicating that a wide range of specific proteins in microglia underlies sensing, activation, and/or other cellular responses. Using RNA sequencing, Hickman et al. (2013) identified 100 transcripts highly enriched in microglia and coined the term "sensome" to describe this class of microglial transcripts ${ }^{8}$. Network analysis of this list identified a TYROBP (for tyrosine kinase binding protein; also known as DAP12, for DNAX activating protein-12)-centered pathway with 24 of these 100 genes interacting directly with TYROBP and 20 interacting indirectly with TYROBP. Concurrently, members of our team employed an integrative network-based approach and identified TYROBP as a key network driver in sporadic late-onset Alzheimer's disease (AD) ${ }^{9}$. More recently, Keren-Shaul et al. ${ }^{10}$ used single-cell RNA sequencing in mouse to define a specific microglial phenotype that they termed "DiseaseAssociated Microglia" (DAM). Tyrobp was one of the genes most robustly upregulated in the proposed earliest stage of transition of microglia from the basal "homeostatic state" into the DAM phenotype.

TYROBP is a transmembrane signaling polypeptide that contains an immunoreceptor phosphotyrosine-based activation motif (ITAM) in its cytoplasmic domain. TYROBP is expressed in microglia and serves as an adaptor for a variety of immune receptors, including two molecules closely linked to AD pathogenesis: TREM2 (triggering receptor expressed on myeloid cells 2) and CR3 (complement receptor 3). TREM2 is expressed at the plasma membrane of microglia in the brain and some mutations and polymorphisms of TREM2 are linked to autosomal dominant AD or sporadic late-onset $\mathrm{AD}^{11}$. Other TREM2 mutations can also cause a polycystic 
leukoencephalopathy osteodystrophy also known by the eponym Nasu-Hakola disease ${ }^{12}$. Most TYROBP mutations represent loss-of-function mutations and also result in Nasu-Hakola disease ${ }^{13}$. Similarly, TYROBP genetic variants have also been identified in early-onset $\mathrm{AD}^{14}$.

TREM2 is required in order for microglia to limit growth of amyloid- $\beta$ (A $\beta$ ) deposits and is essential for a full transition of homeostatic microglia to a DAM state. Keren-Shaul et al. ${ }^{10}$ described a two-stage program for DAM transition with a Trem2-independent step (stage 1) where Tyrobp and other genes are upregulated, followed by a Trem2-dependent step during which both Tyrobp and Trem 2 are upregulated (stage 2). Krasemann et al. ${ }^{15}$ described a very similar microglial signature associated with neurodegenerative diseases, designated the "MGnD" phenotype, and showed that the transition from homeostatic to MGnD microglia was both TREM2- and APOEdependent with a TREM2-APOE signaling pathway driving the transition from homeostatic microglia to MGnD. Notably, when DAM and MGnD are compared, Keren-Shaul et al ${ }^{10}$. also observed by single-cell RNA sequencing an apparent sequence of events whereby Tyrobp was upregulated prior to the upregulation of Trem2. For clarity, since DAM and MGnD microglia appear to share key features of the phenomena described here, we will refer only to DAM for the remainder of this report. Insofar as we are aware, principles established here underpin both DAM and MGnD.

In light of the central role of TYROBP in the microglial sensome ${ }^{8}$, its key role as adaptor for multiple microglial receptors ${ }^{16}$, its upregulation in the early Trem2-independent DAM stage $1^{10}$, and its upregulation in $\mathrm{AD}^{9}$, we employed a number of strategies aimed at interrogation of the causes and consequences of TYROBP upregulation. Using dual RNA in situ hybridization and immunohistochemistry, we found that Tyrobp mRNA level is significantly increased when microglia are recruited, including in wild-type (WT) mouse, in an APP/PSEN1 transgenic mouse model of cerebral $\mathrm{A} \beta$ amyloidosis, and in a $M A P T^{P 301 S}$ transgenic mouse model of tauopathy. To determine whether elevated TYROBP can modify microglial phenotype and AD pathogenesis, we generated a novel transgenic mouse, designated the $I b a 1^{\text {Tyrobp }}$ mouse, wherein the Ibal promoter was used to drive overexpression of a mouse Tyrobp transgene in microglia. The APP/PSEN1 and $M A P T^{P 301 S}$ phenotypes were both altered in the setting of a constitutive increase in TYROBP, as was the transcription of Apoe and associated genes. Finally, using two mouse models of cerebral $\mathrm{A} \beta$ amyloidosis and a mouse model of penetrating cortical stab injury, we show that upregulation 
of Tyrobp and Apoe does not require Trem2, but that upregulation of microglial Apoe requires Tyrobp to reach normal levels.

Our results provide compelling evidence that TYROBP-APOE signaling in the microglial sensome is independent of Trem 2, and we propose that activation of this pathway could be an early or even initiating step in the transformation of microglia from the homeostatic phenotype to the DAM phenotype.

\section{MATERIALS \& METHODS}

\section{Animals}

All experimental procedures were conducted in accordance with the NIH Guidelines for Animal Research and were approved by the Institutional Animal Care and Use Committee (IACUC) at Icahn School of Medicine at Mount Sinai. TYROBP-overexpressing mice, named after Iba1 $1^{\text {Tyrobp }}$ mice, were constructed by cloning the mouse Tyrobp and Egfp sequences separated by an internal ribosome entry site (IRES) under the mouse Ibal promoter in a pBluescript II SK(+) vector ${ }^{17}$. GFP protein was not detected by immunohistochemistry. Microinjection were performed in C57BL/6J mice by Dr Kevin Kelly here at Mount Sinai. APPKM670/671NL/PSEN1 $1^{\text {4exon9 }}$ (APP/PSEN1) and $M A P T^{P 301 S}$ (PS19) mice were obtained from Jackson Laboratories. Tyrobp knockout (Tyrobp ${ }^{-/}$) mice were obtained from Taconic/Merck Laboratory. TgCRND 8 were obtained from Dr Paul Fraser. Trem 2 knockout $\left(\right.$ Trem $\left.2^{-/}\right)$mice were constructed by use of targeted homologous recombination to remove exons 1 and 2, thereby deleting the start codon and the major extracellular IgG domain. In contrast to the reported Velocigene construct, the direction of the Hygromycin cassette was "reversed". Crucially, in agreement with two other models, but in contrast to the Velocigene construct, RT-qPCR analyses confirmed (data not shown) specific loss of Trem2 expression without the perturbation of Treml1 expression observed in the Velocigene construct ${ }^{18}$. For the penetrating cortical stab-injured mice, WT, Tyrobp ${ }^{-/-}$and Trem $2^{-/-}$mice were anesthetized by an intraperitoneal injection of ketamine/xylazine $(80 / 16 \mathrm{mg} / \mathrm{kg}$ body weight) and placed in a stereotactic frame (Stoelting, Wood Dale, IL, USA). The skull was drilled and an empty Hamilton syringe was introduced for 1 minute at the following coordinates (relative to bregma): 
anteroposterior, $-0.3 \mathrm{~mm}$; mediolateral, $\pm 3 \mathrm{~mm}$; dorsoventral, $-2 \mathrm{~mm}$. Mice were killed 3 days after.

\section{Tissue collection and sample preparation}

Mice were anesthetized in a $\mathrm{CO}_{2}$ chamber then transcardially perfused with $20 \mathrm{ml}$ ice-cold phosphate buffered saline (PBS). One hemisphere was post-fixed by incubation for $48 \mathrm{~h}$ in $4 \%$ paraformaldehyde (PFA) and cut into $35 \mu \mathrm{m}$ sections with a vibratome (Leica) for histological analyses (brains used for RNAscope were perfused with both PBS and 4\% PFA prior post-fixation and cut in $10 \mu \mathrm{m}$ sections with a cryostat). The contralateral hemisphere was dissected for isolation of the hippocampus and cortex. Hippocampus was used for RT-qPCR and RNA sequencing, whereas cortical samples were homogenized in a RIPA buffer (Thermo Scientific, \#89901) containing phosphatase and protease inhibitors (Thermo Scientific, \#78442), centrifuged for 20 $\min$ at $15,000 \times \mathrm{g}$ and the supernatant was used.

\section{Immunohistochemistry}

Sections were washed with $0.1 \%$ Triton in PBS, non-specific binding was eliminated by incubation with $0.1 \%$ Triton in PBS $/ 5 \%$ goat serum, and then each slice was incubated with one of a panel of primary antibodies as follows: AT8 anti-pTAU pSer202/Thr205 (1:1000, Thermo Scientific, \#MN1020); anti-pTAU Thr205 (1:1000, Invitrogen, \#44-738G), anti-IBA1 (1:1000, Wako, \#01919741), anti-CD68 (1:500, Bio-Rad, \#MCA1957); anti-6E10 (1:1000, Covance, \#9320500), antiTYROBP (1:500, antibody provided in collaboration with Richard W. Cho at Cell Signaling Technology). For fluorescent immunostaining, sections were incubated with the appropriate secondary antibody: anti-rabbit IgG Alexa Fluor 488 or 568 (1:2000, Invitrogen); anti-mouse IgG Alexa Fluor 488 or 568 (1:2000, Invitrogen); anti-mouse IgG Alexa 350 (1:2000, Invitrogen). For immunoperoxidase immunostaining, endogenous peroxidase was quenched with pre-incubation with PBS containing 3\% $\mathrm{H}_{2} \mathrm{O}_{2}$ for $15 \mathrm{~min}$. Sections were then incubated with the $\mathrm{ABC}$ system (Vectastain Elite ABC HRP Kit, Vector Laboratories, \#PK6100). Horseradish peroxidase conjugates and 3,3'-diaminobenzidine were used for visualization of immune complexes according to the manufacturer's manual (SeraCare, \#5510-0031). Images were obtained with an Olympus BX61 microscope and analyzed with Fiji software (ImageJ). For Thioflavin-S (ThioS) labeling, sections were first mounted on slides and rehydrated prior to incubation with freshly prepared 1\% 
Thioflavin-S (Sigma-Aldrich, \#T1892) for 10 minutes at room temperature, under standard photoprotection conditions. Sections were washed $2 \times 3$ min with $80 \%$ ethanol, then $2 \times 3$ min with $95 \%$ ethanol. The final washes were performed in water $3 \times 5$ min per wash, and coverslips were placed prior to visualization.

\section{Cell culture}

Murine primary microglia were isolated from cerebral cortices, dissected from postnatal day P0P3 WT mice (C57BL/6 J background). Briefly, tissue was homogenized in ice-cold PBS then centrifuged at $300 \mathrm{x}$ g for $5 \mathrm{~min}$. The pellet was resuspended in DMEM supplemented with 10\% heat-inactivated FBS (Gibco), 2 mM glutamine and penicillin/streptomycin (100 U/ml and 0.1 $\mathrm{mg} / \mathrm{ml}$ respectively) and cells were seeded in poly-L-lysine T75 precoated flasks. Cultures were maintained at $37^{\circ} \mathrm{C}$ in humidified $95 / 5 \%$ air $/ \mathrm{CO}_{2}$ incubators for 2 weeks, during which time, media change was performed twice a week. After 2 weeks, cultures were agitated at $180 \mathrm{rpm}$ for $30 \mathrm{~min}$ to detach microglial cells from the astrocytic monolayer for collection. Isolated primary microglia were seeded at 500,000 cells per well ( 24 wells plate) and treated one day after with $2 \mu \mathrm{g} / \mathrm{ml}$ of Lipopolysaccharide (LPS, Sigma, \#L3024-5MG) for 1h. Post-LPS treatment, cells were washed with PBS and RNA was extracted.

\section{RNA in situ hybridization}

Mice used for RNA in situ hybridization (RNAscope ${ }^{\circledR}$ ) were anesthetized in a $\mathrm{CO}_{2}$ chamber and transcardially perfused with $20 \mathrm{ml}$ ice-cold PBS followed by $20 \mathrm{ml}$ ice-cold 4\% paraformaldehyde (PFA). Brains were then post-fixed in $4 \%$ PFA for $24 \mathrm{~h}$ at $4^{\circ} \mathrm{C}$ followed by equilibration in several sucrose gradients $(10 \%$, then $20 \%$, finally $30 \%)$. Tissue samples were embedded in optimal cutting temperature compound (OCT), frozen on dry ice, and $10 \mu \mathrm{m}$-thick sections were cut using a cryostat (Leica). Fluorescent in situ hybridization (FISH) was performed using RNAscope ${ }^{\circledR}$ according to the manufacturer's instructions (ACD). Briefly, the mounted frozen sections were washed in PBS then baked at $60^{\circ} \mathrm{C}$ for $30 \mathrm{~min}$ (Lab-Line Instruments Inc., Melrose Park, IL, USA). Slides were then postfixed in $4 \%$ PFA for $1 \mathrm{~h}$ at room temperature (RT). After fixation, the sections were dehydrated in ethanol (incubated serially in 50\%, then $70 \%$, then $100 \%$ ethanol for 5 min at each concentration) and permitted to dry at RT for $45 \mathrm{~min}$. Sections were treated with $\mathrm{H}_{2} \mathrm{O}_{2}$ for 10 min and washed twice with distilled water. Subsequently, target retrieval was performed by boiling 
the slides for $5 \mathrm{~min}$ in retrieval reagent. Slides were then washed in distilled water, immersed in $100 \%$ ethanol, and finally subjected to air-drying for $5 \mathrm{~min}$ at RT. Sections were then treated with protease III for $20 \mathrm{~min}$ at $40^{\circ} \mathrm{C}$ in the pre-warmed ACD HybEZ II Hybridization System (Cat. No. 321721, ACD) inside the HybEZ Humidity Control Tray (Cat. No. 310012, ACD). After this step, sections were washed twice with distilled water. The following probes from ACD were used and diluted at 1:50 in the RNAscope ${ }^{\circledR}$ Probe Diluent (Cat No. 300041): Tyrobp-C3 (NM_011662.2, bp8-580, Cat. No. 408191-C3), Trem2-C2 (NM_031254.3, bp2-1081, Cat. No. 404111-C2) and Apoe-C4 (NM_009696.3, bp83-1245, Cat. No. 313271-C4). Sections were then hybridized with one corresponding probe at $40^{\circ} \mathrm{C}$ for $2 \mathrm{~h}$ in the HybEZ Oven (ACD), washed twice with $1 \mathrm{X}$ wash buffer, and stored overnight at RT in 5x SSC buffer (Thermo Fisher Scientific, Fair Lawn, NJ, USA). The next day, slides were rinsed $2 \times 2$ min with wash buffer ( 2 min per wash), followed by the three amplification steps (AMP 1, AMP 2, and AMP 3 at $40^{\circ} \mathrm{C}$ for 30, 30, and $15 \mathrm{~min}$ respectively, with two washes with wash buffer after each amplification step). The signal was developed by treating the sections with the HRP reagent corresponding to each probe channel (e.g. HRP-C2, HRP-C3 or HRP-C4) at $40^{\circ} \mathrm{C}$ for $15 \mathrm{~min}$, followed by the TSA Plus fluorophore Opal 690 (dilution of 1:750 in RNAscope ${ }^{\circledR}$ Multiplex TSA Buffer [Cat. No 322809]), at $40^{\circ} \mathrm{C}$ for 30 min, and HRP blocker at $40^{\circ} \mathrm{C}$ for $15 \mathrm{~min}$, with two wash steps after each of the incubation steps. Finally, the slides were counterstained or not with DAPI for 30 seconds. Fluorescent immunohistochemistry was performed by incubating the slides with the primary antibody diluted in PBS for $2 \mathrm{~h}$ at RT. Slides were washed twice for 5 min per wash with $1 \mathrm{X}$ wash buffer, and then incubated with the secondary antibody diluted in PBS for 1h30. Immunostained slides were then washed twice with $1 \mathrm{X}$ wash buffer and mounted.

\section{Western blotting}

Equal amounts of protein from (30 $\mu \mathrm{g}$, mouse cortex was used) were separated by electrophoresis in precast $4-12 \%$ Bis-Tris Gels (Bio-Rad) and transferred to nitrocellulose membranes. The membranes were hybridized with the following primary antibodies as indicated: TYROBP (1:500, antibody provided in collaboration with Dr Richard W. Cho at Cell Signaling Technology); AT8 anti-pTAU pSer202/Thr205 (1:1000, Thermo Scientific, \#MN1020); anti-GAPDH (1:2000, Santa Cruz, \#SC-32233), anti-p-TAU Thr205 (1:1000, Invitrogen, \#44-738G), anti-TAU HT7 (1:1000, Invitrogen, \#MN1000). Secondary antibodies included: peroxidase-labeled anti-mouse IgG 
(1:2000, Vector Laboratories); peroxidase-labeled anti-rabbit IgG (1:2000, Vector Laboratories); peroxidase-labeled anti-rat IgG (1:2000, Vector Laboratories). ECL (Pierce, \#32106) was used to reveal the immunoreactive proteins, and images were acquired using a Fujifilm ImageReader LAS4000. Membranes were stripped using a stripping buffer (Thermo Scientific, \#46430) when required. Luminescent immunoreactive protein bands were quantified using Fiji software (ImageJ).

\section{A $\beta$ assays}

To quantify A $\beta$ levels, human/rat A $\beta$ 1-40/1-42 ELISA kits (Wako, \#294-64701, \#290-6260) were used according to the manufacturer's instructions. Absolute concentrations of A $\beta$ were normalized to initial tissue weight. Supernatants from the RIPA-extracted cortices were used.

\section{RNA extraction and RT-qPCR analysis}

RNAs were isolated from mice hippocampi using the QIAzol Lysis Reagent (Qiagen) and the miRNeasy Micro Kit (Qiagen) following the manufacturer instructions. For the qPCR analyses, 1 $\mu \mathrm{g}$ of RNA was reversed transcribed using the High Capacity RNA-to-cDNA kit (ThermoFisher, \#4387406). The All-in-One qPCR Mix (GeneCopoeia, \#QP001-01) was used to perform RTqPCR. 40 cycles were done and the abundance of each transcript was normalized to the abundance of GAPDH with the $\Delta \Delta \mathrm{Ct}$ method. The sequences of oligonucleotides used can be found in Litvinchuk et al. $2018^{19}$ except for mouse $I L 6$ and TGF $\beta$ where TaqMan ${ }^{\circledR}$ probes were used.

\section{RNA sequencing}

RNA sequencing on mice hippocampi was performed by Novogene (https:// en.novogene.com) using Illumina Novaseq 6000 S4 flow cells. Only samples with RNA integrity number (RIN) $>9$ were included. Non-directional libraries were constructed with a NEB kit using the manufacturer's protocol. RNA sequencing assays were performed after ribosomal RNA depletion by Ribo-Zero. For the data QC, four main steps were implemented including determination of the (1) distribution of sequencing quality; (2) distribution of sequencing error rate; (3) distribution of A/T/G/C bases; and (4) results of raw data filtering. The filtering process included: (1) removal of reads containing adapters, (2) removal of reads containing $\mathrm{N}>10 \%$ ( $\mathrm{N}$ represents bases that cannot be determined), and (3) removal of reads containing low quality bases (Qscore $\leq 5$ ) that are over $50 \%$ of the total 
bases contained in the read. Sequencing reads were aligned to mouse reference genome mm10 (GRCm38.90) using STAR aligner guided by mouse GENCODE gene model release v15. Accepted mapped reads were summarized to gene levels using the featureCounts program. Raw count data were normalized by the voom function in the $\mathrm{R}$ limma package, after which differential expression was identified by the moderated t-test implemented in limma. Differentially expressed genes (DEGs) were defined to have at least 1.2-fold change in expression and BenjaminiHochberg adjusted $\mathrm{p} \leq 0.1$ comparing different genotypes.

\section{Statistics}

The non-genomic data were analyzed with GraphPad Prism 8. Graphs represent the mean of all samples in each group \pm SEM. Sample sizes (n values) and statistical tests are indicated in the figure legends. One-way or two-way ANOVA were used for multiple comparisons. A Student's ttest was used for simple comparisons. Significance is reported at $* \mathrm{p}<0.05, * * p<0.01, * * * p<$ 0.001 and $* * * * p<0.0001$. Grubb's test for outliers was used with $\alpha=0.05$.

\section{RESULTS}

\section{Tyrobp transcription is increased in recruited microglia}

Microglia continuously sense changes in the brain environment and are recruited to sites of injury, microbial invasion, or where abnormal folding or modification of cellular constituents are detected, as with the accumulation of aggregated $\mathrm{A} \beta$. We performed dual RNA in situ hybridization (RNAscope ${ }^{\circledR}$ ) and immunohistochemistry for Tyrobp and IBA1 respectively in WT mice and observed increased levels of Tyrobp mRNA in areas exhibiting clustered microglia (Fig 1A). Using the same experimental approach in two independent mouse models of cerebral amyloidosis $\left(A P P / P S E N 1^{20}\right.$ and $\left.5 x F A D^{21}\right)$, we observed a similar pattern in that the Tyrobp mRNA level is extensively and selectively increased in microglia recruited in close proximity to amyloid plaques as compared to microglia that are more distant from the plaques (Fig. 1B-C). We similarly assayed Tyrobp mRNA and IBA1 protein in the MAPT $T^{301 S}$ mice $^{22}$ (also known as PS19), a mutant tauopathy mouse model. We previously described an elevated number of anti-phosphorylatedTAU immunostained neurons in the piriform cortex ${ }^{23}$, and we detected increased amounts of 
Tyrobp mRNA in microglia in this same region (Fig. 1D). We confirmed the increase of TYROBP at the protein level in microglia around amyloid plaques (Fig. 1E) as previously reported ${ }^{24}$. To discriminate between the role of TYROBP in activated vs recruited microglia, we isolated primary microglia from WT mice and exposed them to the gram-negative bacterial endotoxin lipopolysaccharide (LPS) to induce microglial activation ${ }^{25}$, the status of which we established by quantifying the robust increase of Tnf $\alpha$ mRNA following LPS treatment. Interestingly, Tyrobp mRNA level was unchanged, suggesting that Tyrobp transcription may be increased only when microglia are both recruited and activated but not in resident microglia despite evidence that these residents are also activated (Fig. 1F).

\section{Microglia are normal in $I b a 1^{\text {Tyrobp }}$ mice}

To determine whether constitutive elevation of TYROBP via transgenesis may influence microglial phenotype and progression of $\mathrm{AD}$ pathology, we generated a novel transgenic mouse overexpressing Tyrobp selectively in microglia in the central nervous system. We used the mouse Tyrobp and Enhanced Green Fluorescent Protein (EGFP) sequences separated by an Internal Ribosome Entry Site (IRES) under the control of the mouse Ibal regulatory sequences (Supp Fig. $1^{17}$ ). Microinjections were performed in C57BL/6J mice and one line was selected for further use based on expression level of the transgene, now referred to Ibal ${ }^{\text {Tyrobp }}$. We first assessed the overexpression of Tyrobp mRNA by RT-qPCR and measured a $\approx 2.5$-fold increase (Fig. 2A). Despite this elevated mRNA level, western blot analyses of protein extracts from the cortex did not reveal a significant overexpression at the protein level (Fig. 2B). Using combined RNA in situ hybridization and immunohistochemistry for Tyrobp and IBA1, respectively, in mice deficient $\left(\right.$ Tyrobp $\left.^{-/}\right)$, WT or overexpressing (Ibal ${ }^{\text {Tyrobp }}$ ) Tyrobp, we confirmed the 2-fold increase in Tyrobp mRNA in Ibal Tyrobp mice compared to WT (Fig. 2C-D). We observed that only a subset of microglia was overexpressing Tyrobp mRNA in Ibal ${ }^{\text {Tyrobp }}$ mice. This selectivity is likely due to the use of the Ibal promoter in a WT background without extensive microglia activation, thereby also accounting for the lack of a significant increase of TYROBP at the protein level in the resting state. RNA sequencing of hippocampi from Ibal $1^{\text {Tyrobp }}$ mice did not reveal any differentially expressed genes (DEGs) other than Aifl (=Ibal), which is increased due to the inclusion of the first two exons in the transgenic vector (Supp Fig. 1). These data indicate that Iba $1^{\text {Tyrobp }}$ microglia do not display molecular and phenotypic changes in WT mice. 


\section{TYROBP overexpression in microglia decreases amyloid plaque load in $A P P / P S E N 1$ mice}

To assess whether TYROBP overexpression in microglia modulates $\mathrm{A} \beta$ deposition in APP/PSEN1 mice, double-heterozygous APP/PSEN1;Ibal ${ }^{\text {Tyrobp }}$ mice were generated and studied at 4 months of age. We measured a $\approx 50 \%$ decrease of the plaque density in the cerebral cortices of both male and female APP/PSEN1; Ibal ${ }^{\text {Tyrobp }}$ mice compared to sex-matched APP/PSEN1 mice (Fig. 3A-B) in sections stained for amyloid plaques using Thioflavin $\mathrm{S}$ (ThioS). This observation was supported by measuring levels of human $A \beta 42$ and $A \beta 40$ by ELISA, both of which were apparently associated with a trend toward decreases in the cortex with TYROBP overexpression, mostly among male APP/PSEN1;Ibal Trrobp mice (Fig. 3C). There was no genotype-dependent difference in the number of plaque-associated microglia (Fig. 3D-E), unlike what has been reported in $5 x F A D$ mice in the presence of a transgenic increase in TREM $2^{26}$. To evaluate microglia activation, we probed both groups with anti-IBA1 antibody and observed a weaker staining in APP/PSEN1;Ibal ${ }^{\text {Tyrobp }}$ mice (Supp Fig. 2A). We next performed RT-qPCR on a group of microglial genes previously described in homeostatic or activated microglia. There was a significant increase of $A x l$ and $C c l 2$ and a decrease of $C d 68$ and $T g f \beta$ in brains of APP/PSEN1;Ibal ${ }^{\text {Tyrobp }}$ mice (Fig. 3F). Finally, we observed that the decrease of amyloid plaques persisted in 8-month-old APP/PSEN1;Iba1 ${ }^{\text {Tyrobp }}$ mice as shown by the decreased percentage of ThioS positive areas in somatomotor, hippocampus, and visual areas (Fig. 3G-H).

\section{TYROBP overexpression in $M A P T^{P 301 S}$ mice increases TAU phosphorylation and microglia activation}

We previously reported that deletion of Tyrobp altered both mouse amyloid and tauopathy phenotypes and the microglial response to these pathologies ${ }^{23,27,28}$. In MAPT ${ }^{\text {P301S }}$;Ibal ${ }^{\text {Tyrobp }}$ double heterozygous mice, western blot analyses using AT8 and T205 antibodies revealed increased levels of phosphorylated-TAU (pTAU) in the cortex of both male and female mice compared to $M A P T^{P 301 S}$ mice at 4 months of age, whereas total human TAU levels detected with the HT7 antibody were unchanged (Fig. 4A-B). Increased pTAU within brains from MAPT ${ }^{P 301 S}$;Ibal ${ }^{\text {Tyrobp }}$ mice was further confirmed immunohistochemically (Fig. 4C). We also observed increased IBA1 intensity in $M A P T^{P 301 S}$; Iba1 ${ }^{\text {Tyrobp }}$ compared to MAPT $T^{P 01 S}$ mice that correlated with the increased pTAU (Fig. 4D, Supp Fig. 2B). We confirmed an increased microglial activation state by doublelabel immunohistochemistry with anti-IBA1 and anti-CD68 in the piriform cortex (Fig. 4E). Using 
RT-qPCR, we measured increases of Tyrobp, P2ryl2, Apoe, Axl, Itgax, Iba 1, Tgf $\beta$ and Il6 mRNAs in $M A P T^{P 301 S}$; Ibal $1^{\text {Tyrobp }}$ mice compared to $M A P T^{P 301 S}$ mice (Fig. 4F).

\section{Iba1 ${ }^{\text {Tyrobp }}$ mice reveal a likely relationship between Tyrobp and Apoe}

Despite the obvious differences across $A P P / P S E N 1$ and $M A P T^{P 301 S}$ mouse models and the diverse consequences of Tyrobp upregulation in each of these mice, there are shared changes in $\mathrm{Axl}, \mathrm{Ccl}$, $T g f \beta$ and $I l 6$ mRNAs in both APP/PSEN1 or MAPT $T^{P 301 S}$ mice overexpressing TYROBP (Fig. 3F and 4F). These genes have been recently associated with Apoe in microglia, macrophages and mononuclear phagocytes. AXL has been identified as a regulator of $\mathrm{APOE}^{29}$ and accumulation of IL6 and CCL2 have been associated with APOE overexpression ${ }^{30,31}$. Similarly, reciprocal suppression of TGF $\beta$ and induction of APOE have been described in DAM microglia ${ }^{15}$. Apoe is indeed significantly upregulated in $M A P T^{P 301 S}$;Ibal ${ }^{\text {Tyrobp }}$ mice as compared to $M A P T^{P 301 S}$ mice but not in APP/PSEN1;Ibal ${ }^{\text {Tyrobp }}$ mice compared to APP/PSEN1 mice (Fig. 3F and 4F). However, in bulk RNA sequencing on hippocampi from male APP/PSEN1;Ibal ${ }^{\text {Tyrobp }}$ vs APP/PSEN1 mice, we identified Apoe as a potential (activation z-score: 2.44; p-value overlap: 0.224) upstream regulator (Supp Fig. 3), suggesting the possible existence of a relationship between Tyrobp upregulation and Apoe. While a TREM2-APOE pathway has been described ${ }^{15}$, it is interesting to note that these data support the possibility that the TYROBP-APOE relationship is detectable even in the absence of Trem2 upregulation (Fig. 3F and 4F).

\section{Induction of microglial Tyrobp and Apoe is Trem2-independent in a model of cortical stab injury}

To assess the interactions amongst Trem2, Tyrobp and Apoe in microglia, we used an injury paradigm by introducing a small penetrating cortical stab injury via stereotactic surgery into one hemisphere of the mouse brain in order to induce a recruitment of microglia around the injury site $^{32}$. We first used injured- WT mice and combined RNA in situ hybridization and immunohistochemistry for Apoe and IBA1 respectively. In the intact hemisphere, most Apoe mRNA was not located in microglia but rather in astrocytes, the source of most APOE in brain. However, Apoe mRNA was dramatically increased in microglia recruited on the lesioned side (Fig. 5A). Following the same procedure in Tyrobp ${ }^{-/}$mice, Apoe mRNA was not induced in microglia on either side (Fig. 5B), but strikingly, mRNA levels of Tyrobp and Apoe were highly upregulated 
in the recruited microglia of injured Trem $2^{-/}$mice (Fig. 5C, Supp Fig. 4). Taken together, these data indicate that Tyrobp upregulation in recruited microglia around the traumatic lesion is Trem2independent. Moreover, the increase of Apoe transcripts in recruited microglia in the same mouse model of injury appears to be Tyrobp-dependent and Trem2-independent.

\section{Induction of microglial Tyrobp and Apoe around amyloid plaques is Trem 2-independent, and Apoe upregulation is dramatically decreased when Tyrobp is absent}

In order to investigate further these interactions among Trem2, Tyrobp and Apoe in microglia in the presence of mutant human $A P P$, we first performed dual RNA in situ hybridization and immunohistochemistry for Tyrobp, IBA1 and 6E10 in TgCRND8 mice ${ }^{33}$ on either a WT or Trem2null background. Despite reduced recruitment of microglia around the plaques when Trem2 is deleted $^{24}$, Tyrobp mRNA was still increased in plaque-associated microglia (Fig. 6A). as was Apoe mRNA in plaque-associated microglia in the same TgCRND8;Trem2 $2^{-/-}$mice (Fig. 6B). We then assayed APP/PSEN1 mice that were either WT or deficient for Tyrobp and, while the expression of Apoe was not completely abolished by deletion of Tyrobp, we confirmed a substantial decrease in the induction of Apoe mRNA in plaque-associated microglia when Tyrobp was absent (Fig. 6C).

\section{DISCUSSION}

TREM2, TYROBP and APOE are three microglial genes linked in a pathway contributing to the pathogenesis of $\mathrm{AD}$ and in the transition to $\mathrm{DAM}^{10}$. TYROBP was identified as a key driver in late onset $\mathrm{AD}^{9}$. TREM2 binds to TYROBP, its intracellular adaptor, to initiate its signal transduction pathway, and naturally occurring loss-of-function mutations of either TYROBP or TREM2 can lead to Nasu-Hakola disease $\mathrm{e}^{13,34}$. There is a general assumption among investigators in this research area that genetic deletion or overexpression of either TREM2 or TYROBP would result in identical phenotypes in disease models, but, until now, this has not been tested directly. We previously demonstrated amelioration of behavior and electrophysiologic impairments in APP/PSEN1 and MAPT $T^{P 01 S}$ mice on a Tyrobp-null background, despite a concurrent absence of effect on amyloid pathology and an apparent increase in the stoichiometry of phosphorylated TAU vs total $\mathrm{TAU}^{23,27,28}$. Homozygous deletion of Trem2 can also lead to amelioration of both 
amyloidosis and tauopathy ${ }^{35,36}$, but those effects vary according to the mouse model, and the age and level of deficiency at sacrifice ${ }^{23,37,38}$. Lee et al. ${ }^{26}$ used bacterial artificial chromosome (BAC)mediated transgenesis to overexpress the human TREM2 in the mouse genome and showed that TREM2 overexpression reduces amyloid accumulation in 5xFAD mice. Using a similar BAC system, Gratuze et al. ${ }^{39}$ assessed the impact of TREM2 $2^{R 47 H}$ in $M A P T^{P 301 S}$ mice but no TREM2 overexpression was reported in that study.

The possible existence of an early TREM2-independent phase in conversion of microglia to DAM was described by Keren-Shaul et al. ${ }^{10}$ but was not evident in studies by either Krasemann et al. ${ }^{15}$ or Zhou et al. ${ }^{40}$ Apoe has also been described as a participant in stage 1 of DAM with Tyrobp ${ }^{10}$ and it has been suggested that it drives the DAM transition through a TREM2-APOE pathway ${ }^{15}$. Moreover, Apoe has been reported to influence both amyloidosis and tauopathy histological phenotypes in mouse models ${ }^{41,42}$. A complete elucidation of the choreography of the regulatory interactions among these genes and their cognate proteins therefore remains an area of intense interest. We would suggest that the discrepancies across the various analyses might be explained in part by the fact that DAM microglia are located in the immediate proximity of the plaques, and that neither bulk- nor single-cell- RNA sequencing can distinguish homeostatic vs DAM phentoypes since both techniques generate an average transcriptome analysis from all microglia in a given tissue sample. This formulation played a major role in prompting us to use dual RNA in situ hybridization and immunohistochemistry in the current study in which we sought to determine 1) the effects of transgenic overexpression of Tyrobp on amyloid and tau pathologies and 2) the relationship of the induction of Tyrobp to these pathologies and to the induction of Trem2 and Apoe.

While this manuscript was in preparation, Chen et al. (2020) reported spatial transcriptomics and in situ sequencing in $A p p^{N L-G-F}$ mice to avoid the averaging of the transcriptome in a tissue sample. These investigators proposed the formulation of a plaque-induced gene (PIG) network in microglia and astrocytes in immediate proximity to amyloid plaques with differential expression of 57 genes $^{43}$. Top genes highly upregulated in the proximity of the plaques and as early as 3 months of age were Tyrobp, Apoe and several complement-related genes. Notably, Tyrobp is a key regulator of the complement subnetwork ${ }^{9}$ and $\mathrm{Clq}$ is down-regulated in $A P P / P S E N 1$ and $M A P T^{P 301 S}$ mice 
in the absence of Tyrobp ${ }^{23,28}$. The authors performed the same type of experiment in human AD brain slices and confirmed the enrichment of Tyrobp and several complement components (ClqA, $C l q B, C l q C$, and $C l u$ ). Of particular relevance to our data herein, Trem2 was not included in the human PIG network. Furthermore, Srinivasan et al. ${ }^{44}$ recently used RNA sequencing to profile fluorescence-activated cell sorted (FACS)-purified human microglia from frozen AD and control brains and showed that one of the top gene upregulated in human-AD microglia was Apoe, confirming potential importance of the interplay between Tyrobp and Apoe.

With the inclusion of Tyrobp as a PIG gene ${ }^{43}$ and because of our prior validation of its actions as a driver of $\mathrm{AD}^{9,23,27,28}$, we hypothesized that constitutive overexpression of microglial Tyrobp via transgenesis would alter both amyloid and tau pathologies. In APP/PSEN1 mice, Tyrobp upregulation decreased the amyloid burden, similar to what occurs with the upregulation of Trem $2^{26}$ and supported by a recent report showing that higher microglia activation shows protective effects on subsequent amyloid accumulation ${ }^{45}$. In $M A P T^{P 301 S}$ mice, we previously reported that deficiency of Tyrobp increased TAU phosphorylation and $\operatorname{spread}^{23}$, and were puzzled, therefore, to observe a similar increase in TAU phosphorylation in the MAPT $T^{301 S}$ mice with overexpression of Tyrobp. MAPT $T^{P 301 S}$;Ibal ${ }^{\text {Tyrobp }}$ microglia are more reactive than $M A P T^{P 301 S}$ microglia, and reactive microglia have been reported to drive TAU pathology, so these two observations are compatible $\mathrm{e}^{46,47}$. This exacerbation of pathology under conditions of either downor up-regulation of Tyrobp in MAPT $T^{P 301 S}$ mice reveals the complexity of the microglial events underpinning tauopathy. Our formulation is that, for any particular microglial activation status, there exists some optimum level of Tyrobp expression, and that either elevation or deficiency of Tyrobp levels can be detrimental. These data also support a key role for microglial TYROBP in $\mathrm{AD}$ pathology progression, as we previously proposed in our reports on mice deficient for Tyrobp $23,27,28$.

Our data indicate that Tyrobp upregulation is an early marker of recruited microglia and can occur even in the brains of Trem2-deficient mice. Similarly, we observed that the increased Apoe mRNA level in microglia is Trem2-independent, whether in injury or AD-related mouse models. Finally, we observed that microglial Apoe mRNA level was greatly attenuated in plaque-associated microglia in Tyrobp-deficient mice. These data confirm the model proposed by Keren-Shaul et 
$a{ }^{10}$ in which Tyrobp and Apoe transcripts are increased first, and neither transcription event requires the presence of Trem2. Moreover, Meilandt et al. ${ }^{48}$ recently reported that microglial APOE expression was not reduced, but, on the contrary, was increased in PS2APP;Trem2 $2^{-/}$when compared with microglial APOE expression in PS2APP mice. In that same study, they also analyzed the expression profiles of FACS-purified microglia from $5 x F A D$ mice either in the presence or absence of Trem2 and showed a two-fold reduction in Apoe in one dataset $(\text { GSE132508) })^{10,48}$ but no reduction at all in the other (GSE65067) $)^{48,49}$. However, Parhizkar et al. ${ }^{50}$ reported that the absence of functional TREM2 reduces plaque-associated APOE. This is in line with what Krasemann et al. ${ }^{15}$ proposed when they showed that genetic targeting of Trem 2 suppresses the APOE pathway. Our observations and conclusions herein apparently differ from those of Parhizkar et al. ${ }^{50}$ to the extent that, in our hands, microglial amyloid-plaque sensing followed by upregulation of Tyrobp and Apoe are preserved despite the absence of Trem 2 and, as a consequence of the Trem 2 deficiency, microglia recruitment into the proximity of amyloid plaques is reduced. This relationship points to the fact that the absence of functional TREM2 will block appearance of the full DAM phenotype and therefore the associated clearance of the plaques is reduced. Nevertheless, we propose a model wherein the sensing of amyloid plaques --which takes place upstream of amyloid plaque clearance-- involves Tyrobp and Apoe but not necessarily Trem2.

Considering the central role of Tyrobp described in the microglial sensome ${ }^{8}$, its upregulation even in the absence of Trem2, and the consequences of its overexpression in APP/PSEN1 and $M A P T^{P 301 S}$ mice, we propose that Tyrobp is one of the key genes upregulated in the switch from the homeostatic phenotype to the DAM phenotype. Taking all the data together, we propose a model in which microglia perceive stimuli and initiate their responses during an early and relatively brief time window, corresponding to what Keren-Shaul et al. ${ }^{10}$ described as stage 1. During this phase, Trem 2 is not required but Apoe will be upregulated presumably as a downstream consequence of Tyrobp upregulation. TYROBP is a 113 amino acid polypeptide with a minimal extracellular region ${ }^{51,52}$, making it unlikely that TYROBP is the sole player in a signal transduction pathway involving both the perception of the environment and the triggering of the switch from homeostatic phenotype to DAM. However, TYROBP is the adaptor for many receptors other than TREM $2^{16}$, and therefore, it is plausible and perhaps likely that other TYROBP receptors could 
play key roles in sensing the deposition of amyloid. For example, numerous SIGLEC proteins (sialic acid-binding immunoglobulin-type lectins) carry a positively charged residue in their transmembrane domain that participates in oligomerization of the SIGLEC with TYROBP. The primary SIGLEC ligand is a sialic acid that accumulates in many pathological conditions including cerebral A $\beta$ amyloidosis ${ }^{53,54}$. Moreover, Siglec-H interacts with TYROBP, and its expression has been reported to be elevated in 5xFAD mice vs $\mathrm{WT}^{26}$. CD33 (SIGLEC-3) is also one of the most abundant SIGLECs in human brain, and genome-wide association studies (GWAS) implicated a polymorphism near $C D 33$ as a genetic risk factor for $\mathrm{AD}^{2,4,55}$. CD33 and TREM2 both interact with TYROBP, either directly (TREM2) or via common intracellular signaling factors (CD33). Griciuc et al. ${ }^{56}$ recently investigated crosstalk between CD33 and TREM2 and proposed that CD33 acts upstream of TREM2. They also showed that Cd33 and Tyrobp expression levels did not change in Trem $2^{-/-}$versus WT microglia. This formulation provides evidence that CD33-TYROBP signaling could occur upstream of the recruitment and upregulation of TREM2.

Rather than the somewhat "Trem2-centric" view of DAM proposed in the existing AD microglia literature ${ }^{15,40,50}$, we propose that Tyrobp plays a central role in an alternative and early pathway in the microglial sensome ${ }^{8}$, even in the absence of any change in Trem 2 levels. The data that we present here document the robust consequences of TYROBP overexpression in both APP/PSEN1 and MAPT $T^{P 301 S}$ mice. We confirm here that upregulation in microglia of both Tyrobp and Apoe constitute interconnected events in microglia sensing of amyloid deposits, and these events take place independently of Trem2. 


\section{AUTHOR CONTRIBUTIONS}

MA, JVHM, SG and MEE designed the study. MA performed the experiments and analyzed the data. JM contributed to the RNA in situ hybridization related experiments. MW and BZ contributed to the RNA sequencing analysis. JKG and PF provided the TgCRND8 mice. MA, SG and MEE wrote the manuscript.

\section{FUNDING}

The study was supported by the National Institute on Aging (U01 AG046170 and R01 AG057907 to MEE, SG and BZ), the Alzheimer's Disease Research Division of the BrightFocus Foundation (grant A2018253F to MA and grant A2016482F to JVHM), the Mount Sinai Alzheimer's Disease Research Center (ADRC P50 AG005138 and P30 AG066514 to Mary Sano, with internal pilot grant awarded to MA).

\section{CONFLICT OF INTEREST}

The authors declare that they have no competing interests. 


\section{REFERENCES}

1. Lambert, J. C. et al. Meta-analysis of 74,046 individuals identifies 11 new susceptibility loci for Alzheimer's disease. Nat. Genet. 45, 1452-1458 (2013).

2. Naj, A. C. et al. Common variants at MS4A4/MS4A6E, CD2AP, CD33 and EPHA1 are associated with late-onset Alzheimer's disease. Nat. Genet. 43, 436-441 (2011).

3. Lambert, J.-C. et al. Evidence of the association of BIN1 and PICALM with the AD risk in contrasting European populations. Neurobiol. Aging 32, 756.e11-15 (2011).

4. Hollingworth, P. et al. Common variants at ABCA7, MS4A6A/MS4A4E, EPHA1, CD33 and CD2AP are associated with Alzheimer's disease. Nat. Genet. 43, 429-435 (2011).

5. Jun, G. et al. Meta-analysis confirms CR1, CLU, and PICALM as alzheimer disease risk loci and reveals interactions with APOE genotypes. Arch. Neurol. 67, 1473-1484 (2010).

6. Lambert, J.-C. et al. Genome-wide association study identifies variants at CLU and CR1 associated with Alzheimer's disease. Nat. Genet. 41, 1094-1099 (2009).

7. Harold, D. et al. Genome-wide association study identifies variants at CLU and PICALM associated with Alzheimer's disease. Nat. Genet. 41, 1088-1093 (2009).

8. Hickman, S. E. et al. The microglial sensome revealed by direct RNA sequencing. Nat. Neurosci. 16, 1896-1905 (2013).

9. Zhang, B. et al. Integrated systems approach identifies genetic nodes and networks in lateonset Alzheimer's disease. Cell 153, 707-720 (2013).

10. Keren-Shaul, H. et al. A Unique Microglia Type Associated with Restricting Development of Alzheimer's Disease. Cell 169, 1276-1290.e17 (2017). 
11. Guerreiro, R. et al. TREM2 variants in Alzheimer's disease. N. Engl. J. Med. 368, 117-127 (2013).

12. Zhou, S.-L. et al. TREM2 Variants and Neurodegenerative Diseases: A Systematic Review and Meta-Analysis. JAD 68, 1171-1184 (2019).

13. Paloneva, J. et al. Loss-of-function mutations in TYROBP (DAP12) result in a presenile dementia with bone cysts. Nat Genet 25, 357-361 (2000).

14. Pottier, C. et al. TYROBP genetic variants in early-onset Alzheimer's disease. Neurobiol. Aging 48, 222.e9-222.e15 (2016).

15. Krasemann, S. et al. The TREM2-APOE Pathway Drives the Transcriptional Phenotype of Dysfunctional Microglia in Neurodegenerative Diseases. Immunity 47, 566-581.e9 (2017).

16. Lanier, L. L. DAP10- and DAP12-associated receptors in innate immunity. Immunological Reviews 227, 150-160 (2009).

17. Tanaka, K. F. et al. Expanding the Repertoire of Optogenetically Targeted Cells with an Enhanced Gene Expression System. Cell Reports 2, 397-406 (2012).

18. Kang, S. S. et al. Behavioral and transcriptomic analysis of Trem2-null mice: not all knockout mice are created equal. Human Molecular Genetics 27, 211-223 (2018).

19. Litvinchuk, A. et al. Complement C3aR Inactivation Attenuates Tau Pathology and Reverses an Immune Network Deregulated in Tauopathy Models and Alzheimer's Disease. Neuron 100, 1337-1353.e5 (2018).

20. Jankowsky, J. L. et al. Mutant presenilins specifically elevate the levels of the 42 residue beta-amyloid peptide in vivo: evidence for augmentation of a 42-specific gamma secretase. Hum. Mol. Genet. 13, 159-170 (2004). 
21. Oakley, H. et al. Intraneuronal beta-amyloid aggregates, neurodegeneration, and neuron loss in transgenic mice with five familial Alzheimer's disease mutations: potential factors in amyloid plaque formation. J. Neurosci. 26, 10129-10140 (2006).

22. Yoshiyama, Y. et al. Synapse loss and microglial activation precede tangles in a P301S tauopathy mouse model. Neuron 53, 337-351 (2007).

23. Audrain, M. et al. Integrative approach to sporadic Alzheimer's disease: deficiency of TYROBP in a tauopathy mouse model reduces $\mathrm{C} 1 \mathrm{q}$ and normalizes clinical phenotype while increasing spread and state of phosphorylation of tau. Mol. Psychiatry (2018) doi:10.1038/s41380-018-0258-3.

24. Yuan, P. et al. TREM2 Haplodeficiency in Mice and Humans Impairs the Microglia Barrier Function Leading to Decreased Amyloid Compaction and Severe Axonal Dystrophy. Neuron 90, 724-739 (2016).

25. Hoogland, I. C. M., Houbolt, C., van Westerloo, D. J., van Gool, W. A. \& van de Beek, D. Systemic inflammation and microglial activation: systematic review of animal experiments. $J$ Neuroinflammation 12, 114 (2015).

26. Lee, C. Y. D. et al. Elevated TREM2 Gene Dosage Reprograms Microglia Responsivity and Ameliorates Pathological Phenotypes in Alzheimer's Disease Models. Neuron 97, 10321048.e5 (2018).

27. Haure-Mirande, J.-V. et al. Deficiency of TYROBP, an adapter protein for TREM2 and CR3 receptors, is neuroprotective in a mouse model of early Alzheimer's pathology. Acta Neuropathol. 134, 769-788 (2017). 
28. Haure-Mirande, J.-V. et al. Integrative approach to sporadic Alzheimer's disease: deficiency of TYROBP in cerebral $A \beta$ amyloidosis mouse normalizes clinical phenotype and complement subnetwork molecular pathology without reducing A $\beta$ burden. Mol. Psychiatry (2018) doi:10.1038/s41380-018-0255-6.

29. Zhao, W. et al. Axl receptor tyrosine kinase is a regulator of apolipoprotein E. Mol Brain 13, 66 (2020).

30. Levy, O. et al. APOE Isoforms Control Pathogenic Subretinal Inflammation in Age-Related Macular Degeneration. J. Neurosci. 35, 13568-13576 (2015).

31. Levy, O. et al. Apolipoprotein E promotes subretinal mononuclear phagocyte survival and chronic inflammation in age-related macular degeneration. EMBO Mol Med 7, 211-226 (2015).

32. Clarke, D., Penrose, M. A., Harvey, A. R., Rodger, J. \& Bates, K. A. Low intensity rTMS has sex-dependent effects on the local response of glia following a penetrating cortical stab injury. Exp. Neurol. 295, 233-242 (2017).

33. Chishti, M. A. et al. Early-onset amyloid deposition and cognitive deficits in transgenic mice expressing a double mutant form of amyloid precursor protein 695. J. Biol. Chem. 276, 21562-21570 (2001).

34. Dardiotis, E. et al. A novel mutation in TREM2 gene causing Nasu-Hakola disease and review of the literature. Neurobiology of Aging 53, 194.e13-194.e22 (2017).

35. Jay, T. R. et al. TREM2 deficiency eliminates TREM2+ inflammatory macrophages and ameliorates pathology in Alzheimer's disease mouse models. The Journal of Experimental Medicine 212, 287-295 (2015). 
36. Leyns, C. E. G. et al. TREM2 deficiency attenuates neuroinflammation and protects against neurodegeneration in a mouse model of tauopathy. Proc. Natl. Acad. Sci. U.S.A. 114, 11524-11529 (2017).

37. Bemiller, S. M. et al. TREM2 deficiency exacerbates tau pathology through dysregulated kinase signaling in a mouse model of tauopathy. Mol Neurodegener 12, 74 (2017).

38. Sayed, F. A. et al. Differential effects of partial and complete loss of TREM2 on microglial injury response and tauopathy. Proc. Natl. Acad. Sci. U.S.A. 115, 10172-10177 (2018).

39. Gratuze, M. et al. Impact of TREM2R47H variant on tau pathology-induced gliosis and neurodegeneration. Journal of Clinical Investigation 10.1172/JCI138179 (2020) doi:10.1172/JCl138179.

40. Zhou, Y. et al. Human and mouse single-nucleus transcriptomics reveal TREM2-dependent and TREM2-independent cellular responses in Alzheimer's disease. Nat Med 26, 131-142 (2020).

41. Shi, Y. et al. Microglia drive APOE-dependent neurodegeneration in a tauopathy mouse model. J. Exp. Med. (2019) doi:10.1084/jem.20190980.

42. Ulrich, J. D. et al. ApoE facilitates the microglial response to amyloid plaque pathology. J. Exp. Med. 215, 1047-1058 (2018).

43. Chen, W.-T. et al. Spatial Transcriptomics and In Situ Sequencing to Study Alzheimer's Disease. Cell S0092867420308151 (2020) doi:10.1016/j.cell.2020.06.038.

44. Srinivasan, K. et al. Alzheimer's Patient Microglia Exhibit Enhanced Aging and Unique Transcriptional Activation. Cell Reports 31, 107843 (2020). 
45. Ewers, M. et al. Higher CSF STREM2 and microglia activation are associated with slower rates of beta-amyloid accumulation. EMBO Mol Med e12308 (2020) doi:10.15252/emmm.202012308.

46. Maphis, N. et al. Reactive microglia drive tau pathology and contribute to the spreading of pathological tau in the brain. Brain 138, 1738-1755 (2015).

47. Asai, H. et al. Depletion of microglia and inhibition of exosome synthesis halt tau propagation. Nat. Neurosci. 18, 1584-1593 (2015).

48. Meilandt, W. J. et al. Trem2 deletion reduces late-stage amyloid plaque accumulation, elevates the $A \beta 42: A \beta 40$ ratio, and exacerbates axonal dystrophy and dendritic spine loss in the PS2APP Alzheimer's mouse model. J. Neurosci. 1871-19 (2020) doi:10.1523/JNEUROSCI.1871-19.2019.

49. Wang, Y. et al. TREM2 lipid sensing sustains the microglial response in an Alzheimer's disease model. Cell 160, 1061-1071 (2015).

50. Parhizkar, S. et al. Loss of TREM2 function increases amyloid seeding but reduces plaqueassociated ApoE. Nature Neuroscience (2019) doi:10.1038/s41593-018-0296-9.

51. Lanier, L. L., Corliss, B. C., Wu, J., Leong, C. \& Phillips, J. H. Immunoreceptor DAP12 bearing a tyrosine-based activation motif is involved in activating NK cells. Nature 391, 703-707 (1998).

52. Tomasello, E. et al. Gene structure, expression pattern, and biological activity of mouse killer cell activating receptor-associated protein (KARAP)/DAP-12. J. Biol. Chem. 273, 3411534119 (1998).

53. Siddiqui, S. S. et al. Siglecs in Brain Function and Neurological Disorders. Cells 8, (2019). 
54. Salminen, A. \& Kaarniranta, K. Siglec receptors and hiding plaques in Alzheimer's disease. J.

Mol. Med. 87, 697-701 (2009).

55. Bertram, L. et al. Genome-wide association analysis reveals putative Alzheimer's disease susceptibility loci in addition to APOE. Am. J. Hum. Genet. 83, 623-632 (2008).

56. Griciuc, A. et al. TREM2 Acts Downstream of CD33 in Modulating Microglial Pathology in Alzheimer's Disease. Neuron 103, 820-835.e7 (2019). 


\section{FIGURES \& LEGENDS}

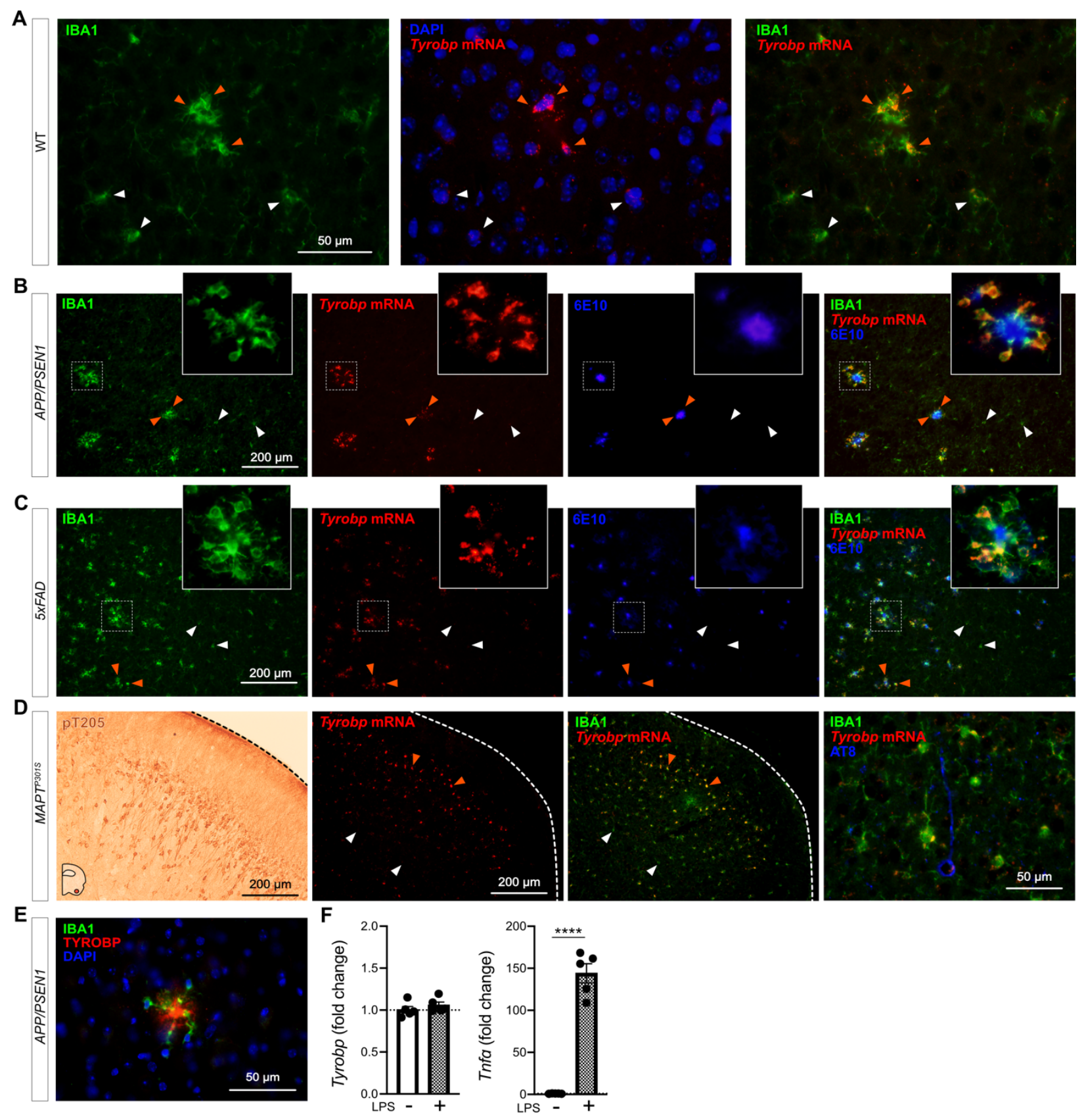

Figure 1: Tyrobp mRNA is increased in recruited microglia

(A) Dual RNA fluorescent in situ hybridization (RNAscope $($ ) and immunohistochemistry for Tyrobp mRNA (red) and IBA1 protein (green) respectively in WT mice (DAPI in blue). Scale bar $=50 \mu \mathrm{m}$. (B-C) Dual RNA in situ hybridization and immunohistochemistry for Tyrobp (red), IBA1 (Green) and $\mathrm{A} \beta$ (antibody 6E10) (blue) in APP/PSEN1 (B) and 5xFAD (C) mice. Scale bar $=200$ 
$\mu \mathrm{m}$. (D) Left panel: representative image of immunohistochemistry with antibody pT205 in the piriform cortex of $M A P T^{P 301 S}$ (PS19) mice. Scale bar $=200 \mu \mathrm{m}$. Right panels: dual RNA in situ hybridization and immunohistochemistry for Tyrobp (red), IBA1 (green) and pTau (antibody AT8) (blue) in the piriform cortex of $M A P T^{P 301 S}$ mice. Scale bars $=200$ and $50 \mu \mathrm{m}$. (E) Coimmunohistochemistry for TYROBP (green) and human $\mathrm{A} \beta$ (antibody 6E10) (red) in APP/PSEN1 mice (DAPI in blue). Scale bar $=50 \mu \mathrm{m}$. (F) RT-qPCR analyses of Tyrobp and TNF $\alpha$ mRNAs in WT primary microglia with and without LPS. Mice were either 4 (A) or 8 (B-E) months of age and were all WT for Tyrobp. White and orange arrows indicate examples of non-recruited and recruited microglia respectively. Slice thickness $=10 \mu \mathrm{m}$. 
A

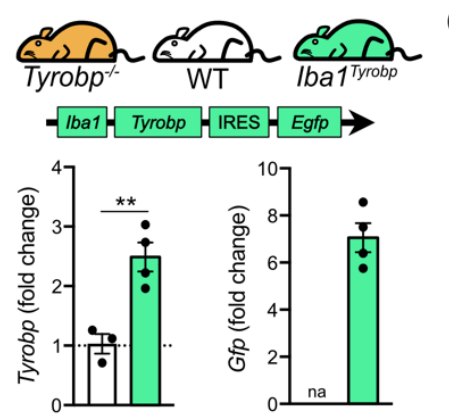

B

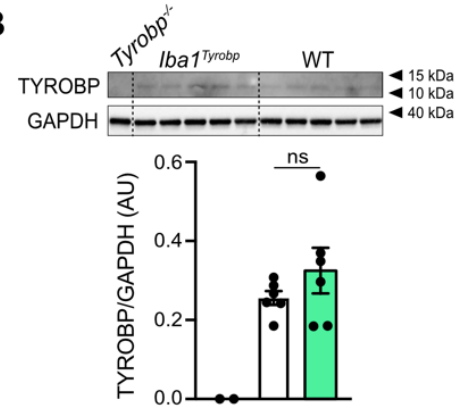

C
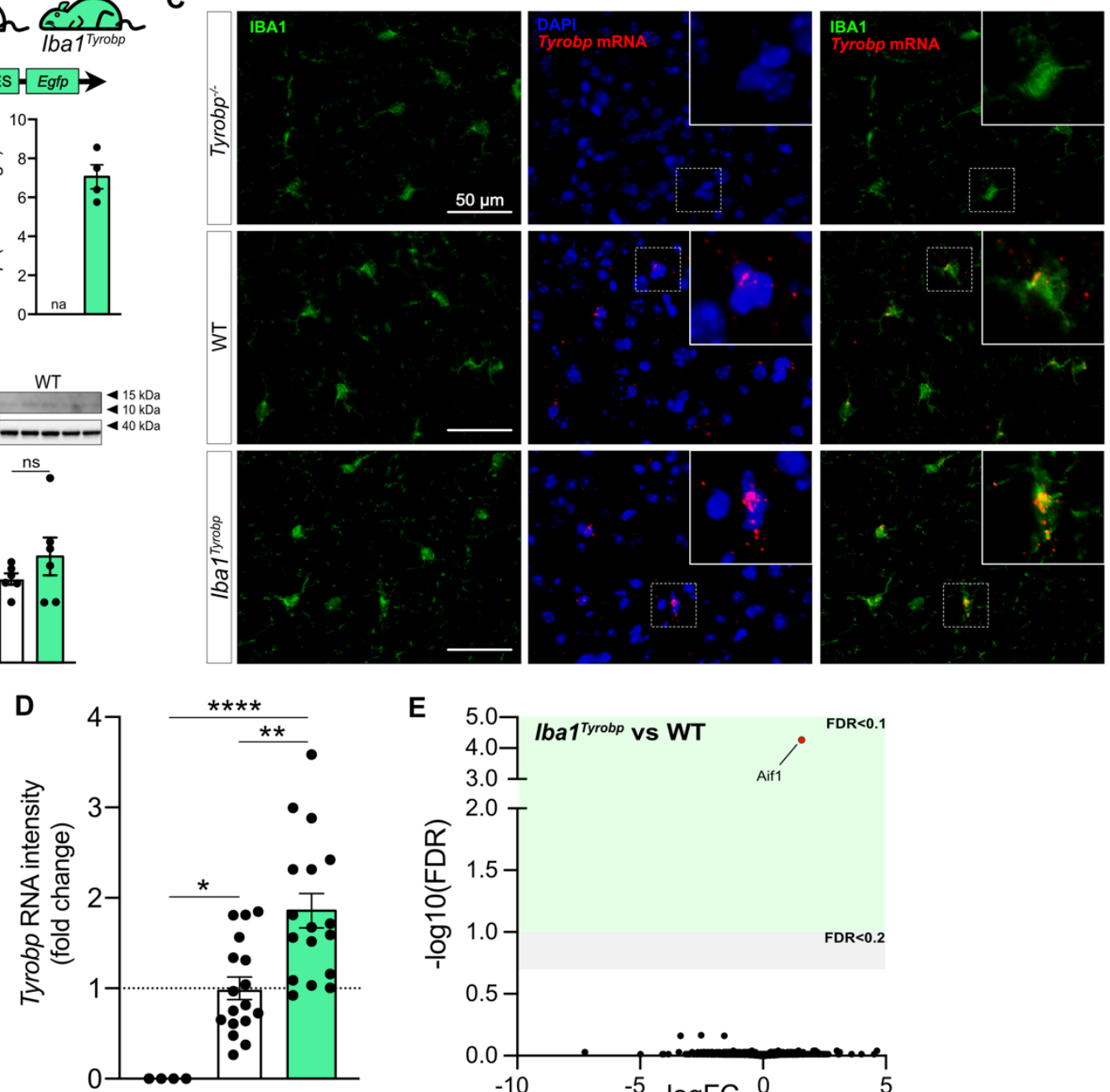

E

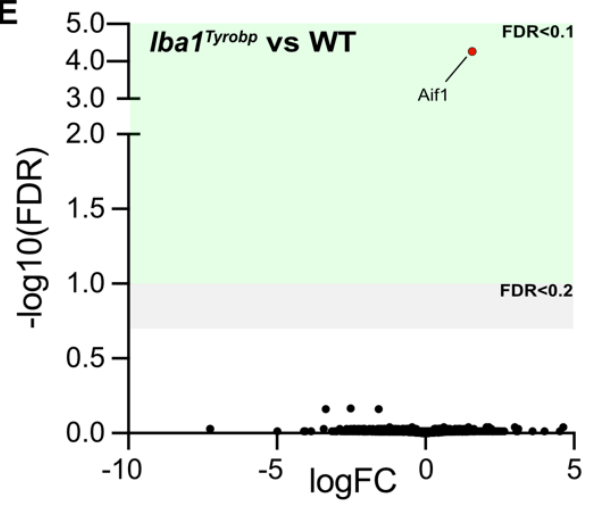

\section{Figure 2: Generation of Iba1 ${ }^{\text {Tyrobp }}$ mice}

(A) Hippocampi from 4-month old Tyrobp ${ }^{-/}$, WT and Ibal Tyrobp mice were assayed for Tyrobp and Gfp mRNAs by RT-qPCR ( $\mathrm{n}=3-4$ mice per group). (B) Representative western blot and quantification of TYROBP and GAPDH in the cortex of the same groups used in (A) (n=2-6 mice per group). (C) Dual RNA fluorescent in situ hybridization and immunohistochemistry for Tyrobp mRNA (red) and IBA1 (green) respectively (DAPI in blue) in Tyrobp ${ }^{-/}$, WT and Ibal ${ }^{\text {Tyrobp }}$ mice. Scale bar $=50 \mu \mathrm{m}$ and slice thickness $=10 \mu \mathrm{m}$. (D) Quantification of Tyrobp mRNA intensity from the experiment described in (C). $n=4,17$ and 17 slices per group (from $N=1$ mouse per genotype) for Tyrobp ${ }^{-/}$, WT and Ibal Tyrobp mice respectively. (E) Volcano plot representation of the whole hippocampal DEGs in Ibal ${ }^{\text {Tyrobp }}$ vs WT mice ( $\mathrm{n}=4$ 4-month old males per genotype). Error bars represent means \pm SEM. Statistical analyses were performed using a Student t-test (A) or a One- 
bioRxiv preprint doi: https://doi.org/10.1101/2020.08.18.254649; this version posted August 19, 2020. The copyright holder for this preprint

(which was not certified by peer review) is the author/funder, who has granted bioRxiv a license to display the preprint in perpetuity. It is made available under aCC-BY-NC-ND 4.0 International license.

Way ANOVA followed by a Tukey's post-hoc test (B, D), ${ }^{*} \mathrm{p}<0.05,{ }^{* *} \mathrm{p}<0.01, * * * * \mathrm{p}<0.0001$. na $=$ not applicable; ns = non-significant. 
A

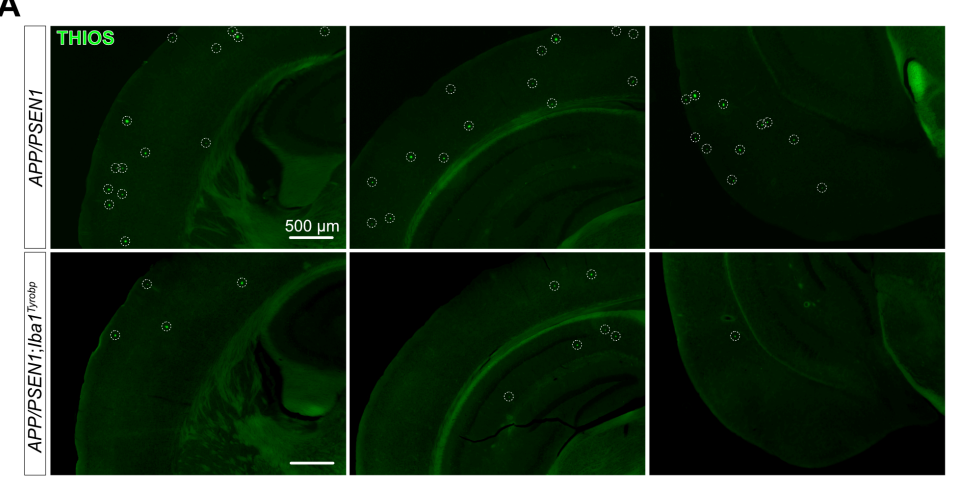

D

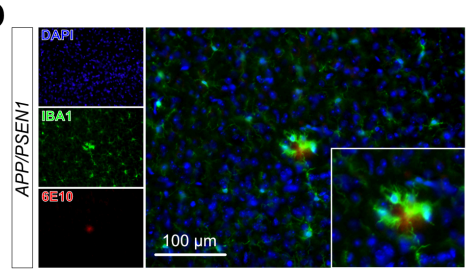

$\mathbf{F}_{20}$
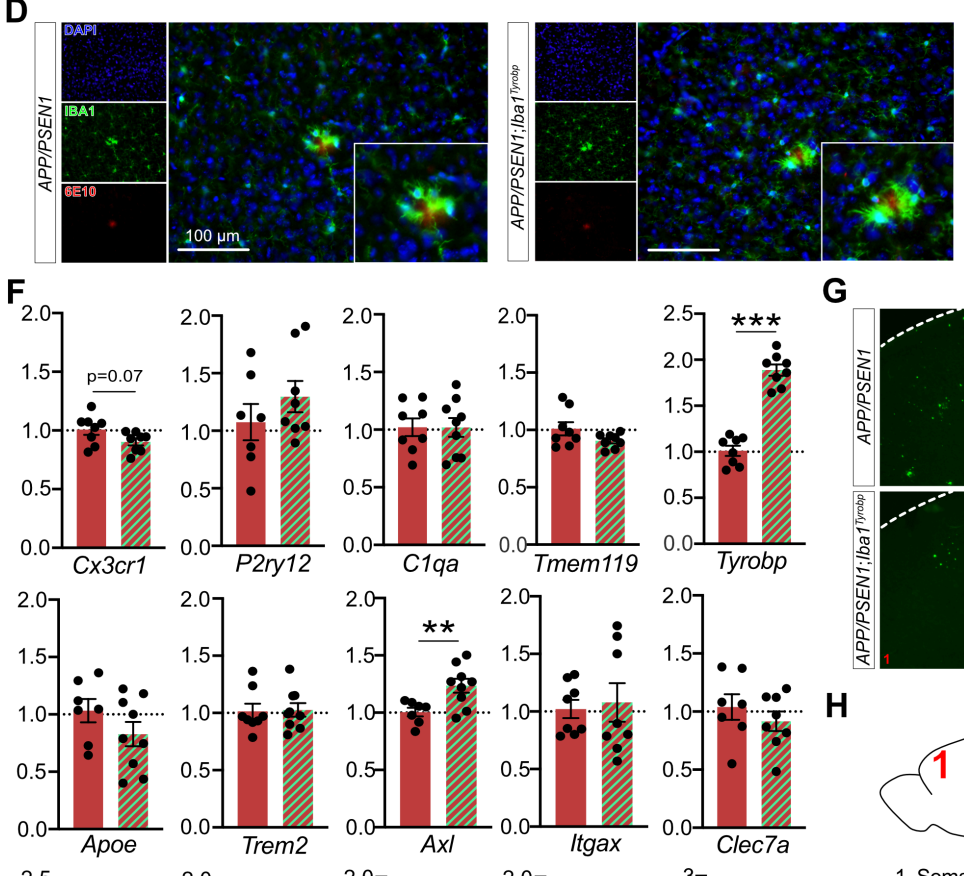

B
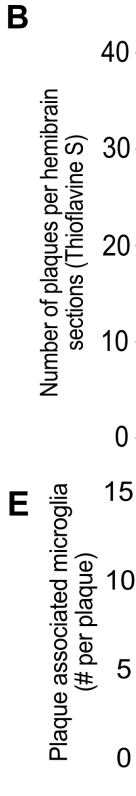

G
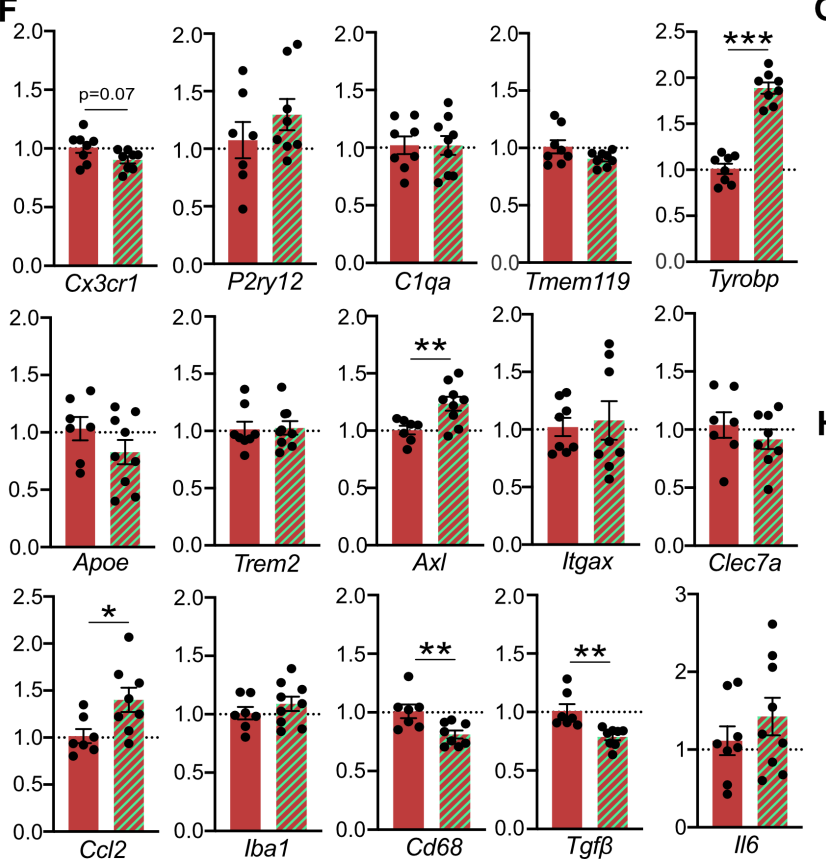
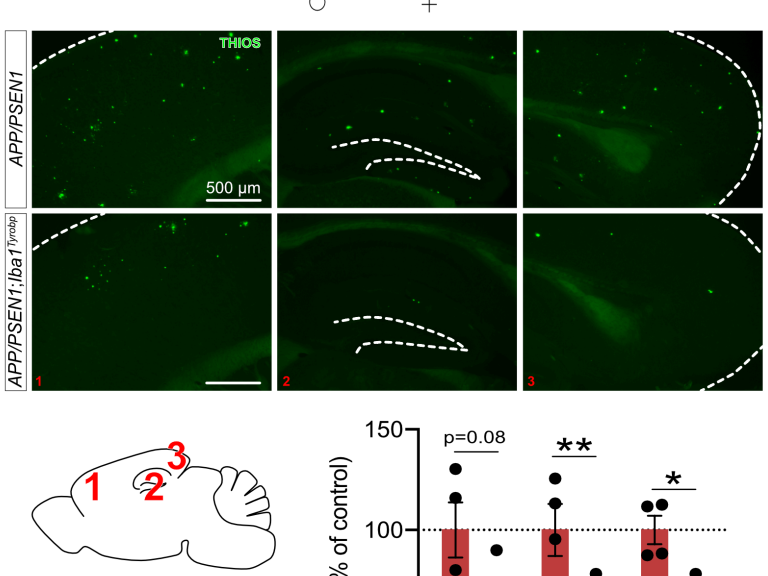

1. Somatomotor areas 2. Hippocampus
3. Visual areas

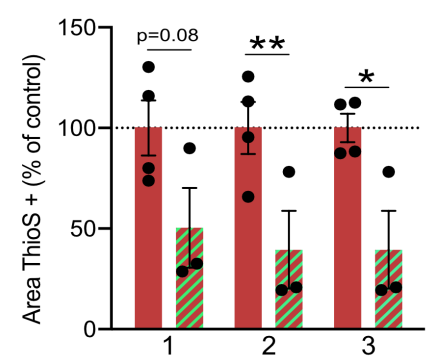

Figure 3: Transgene-derived Tyrobp upregulation decreases amyloid plaque load in APP/PSEN1 mice

(A) Representative images of Thioflavine-S (ThioS) staining in APP/PSEN1 and APP/PSEN1;Iba1 ${ }^{\text {Tyrobp }}$ mice at 4 months of age. Scale bar $=500 \mu \mathrm{m}$. (B) Quantification of the number of ThioS-positive plaques per hemibrain in APP/PSEN1 and APP/PSEN1;Ibal ${ }^{\text {Tyrobp }}$ mice at 4 months of age. $\mathrm{N}=4-5$ mice per genotype and sex with 3 slices per animal. (C) Human $A \beta 42$ and $\mathrm{A} \beta 40$ concentrations measured by ELISA in the cortex of the same groups described in (B). (D) Representative images of double-label immunohistochemistry with anti-IBA1 and anti-6E10 
antibodies in $A P P / P S E N 1$ and $A P P / P S E N 1$; Ibal ${ }^{\text {Tyrobp }}$ mice at 4 months of age. Scale bar $=100$ $\mu \mathrm{m}$. (E) Quantification of the number of plaque-associated microglia in the 4 groups described in (B). $\mathrm{N}=10-24$ plaques from 4-5 mice per group. (F) RT-qPCR analyses of microglial gene mRNAs in the hippocampus of APP/PSEN1 and APP/PSEN1;Ibal ${ }^{\text {Tyrobp }}$ mice at 4 months of age. $\mathrm{N}=7-9$ mice per group, females and males were pooled. $(\mathrm{G})$ Representative images of ThioS staining in male APP/PSEN1 and APP/PSEN1; Ibal ${ }^{\text {Tyrobp }}$ mice at 8 months of age. Scale bar $=500$ $\mu \mathrm{m}$. (H) Quantification of the ThioS immunoreactive area in male APP/PSEN1 and APP/PSEN1;Ibal ${ }^{\text {Tyrobp }}$ mice at 8 months of age (somatomotor and visual areas of the cortex, and hippocampus were quantified). $\mathrm{N}=3-4$ mice per group. Error bars represent means \pm SEM. Statistical analyses were performed using a Two-Way ANOVA followed by a Sidak post-hoc test for $(\mathrm{B}, \mathrm{C}$ and $\mathrm{E})$ or a Student t-test for $(\mathrm{C})$ when $* \mathrm{t}$ is indicated and $(\mathrm{F}-\mathrm{H}),{ }^{*} \mathrm{p}<0.05, *^{*} \mathrm{p}<0.01$, $* * * \mathrm{p}<0.001$. 


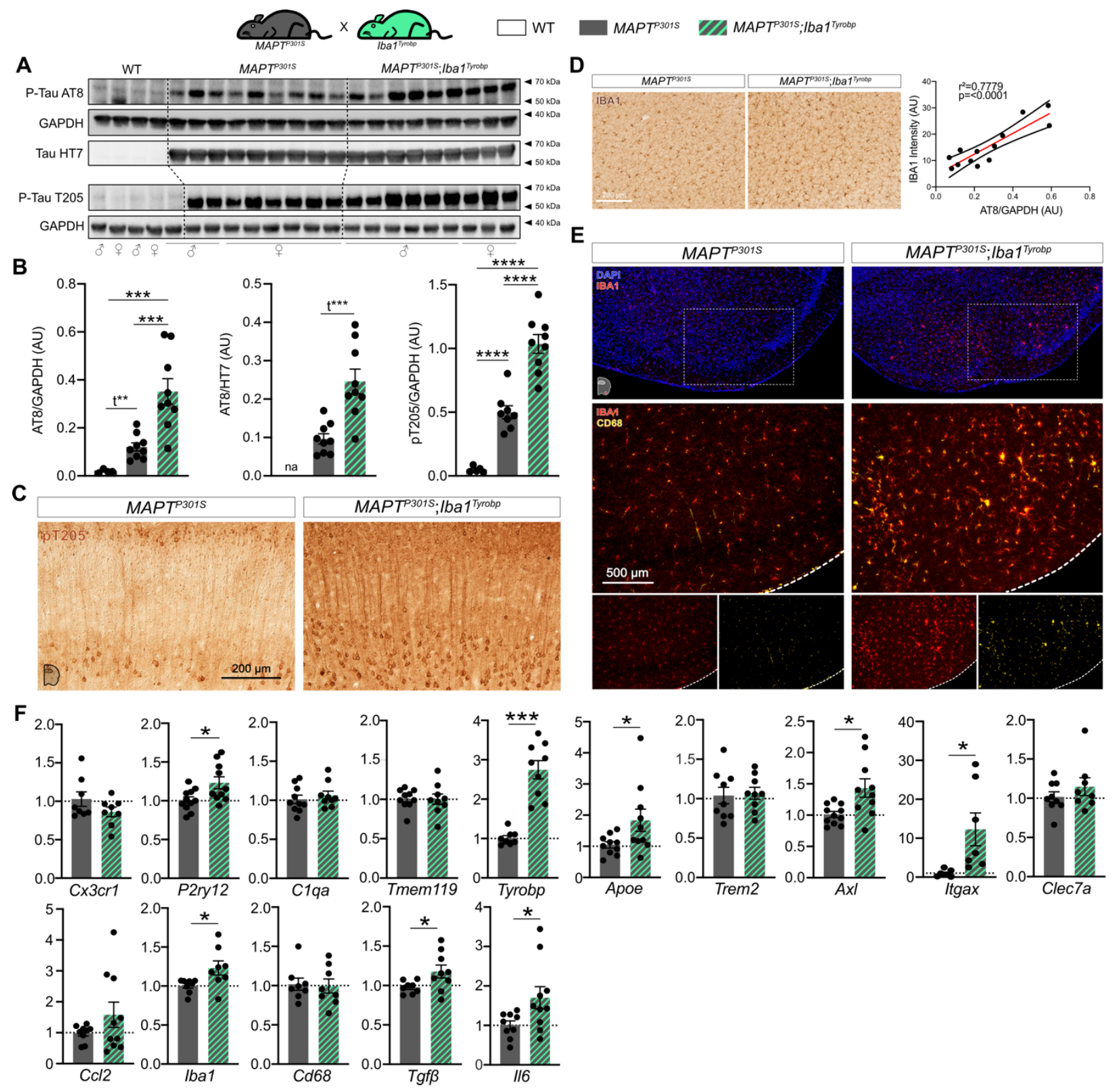

Figure 4: Transgene-induced Tyrobp upregulation increases TAU hyperphosphorylation and microglial activation in 4-month-old $M A P T^{P 301 S}$ mice

(A) Western blot analyses of phosphorylated-TAU on S202 or T205 epitopes (AT8 and pT205 antibodies) and total human TAU (HT7 antibody) in cortical homogenates from WT, MAPT $T^{P 301 S}$ (PS19) and MAPT ${ }^{\text {P301S }} ;$ Ibal $1^{\text {Tyrobp }}$ mice at 4 months-old. $\mathrm{n}=4-9$ mice per group. (B) Densitometric analyses of western blots presented in (A) standardized to GAPDH or HT7. (C) Representative images of DAB-immunohistochemistry with antibody pT205 in 4 month-old $M A P T^{P 301 S}$ and $M A P T^{P 301 S}$;Ibal ${ }^{\text {Tyrobp }}$ mice. Scale bar $=200 \mu \mathrm{m}$. (D) Left panel: representative images of antiIBA1 immunohistochemistry on the same groups described in (C). Scale bar $=200 \mu \mathrm{m}$. Additional 
representative pictures are presented in Supplementary Figure 2. Right panel: western blotAT8/GAPDH quantification plotted against anti-IBA1 immunoreactivity in the cortex. Linear regression with trend line (red line) and 95\% confidence intervals (black lines) are indicated. (E) Representative images of double-label immunofluorescence with anti-IBA1 and anti-CD68 antibodies in the piriform cortex on the same groups described in (C). Scale bar $=500 \mu \mathrm{m}$. (F) RTqPCR analyses of microglial gene mRNAs in the hippocampus of $M A P T^{P 301 S}$ and MAPT ${ }^{\text {P301S }}$;Ibal ${ }^{\text {Tyrobp }}$ mice at 4 months of age. $\mathrm{N}=7-11$ per group. Error bars represent means \pm SEM. Statistical analyses were performed using a One-Way ANOVA followed by a Tukey's posthoc test for (B) or a Student t-test for (B) when $*_{t}$ is indicated and $(\mathrm{F}),{ }^{*} \mathrm{p}<0.05, * * \mathrm{p}<0.01$, $* * * p<0.001, * * * * p<0.0001$. 


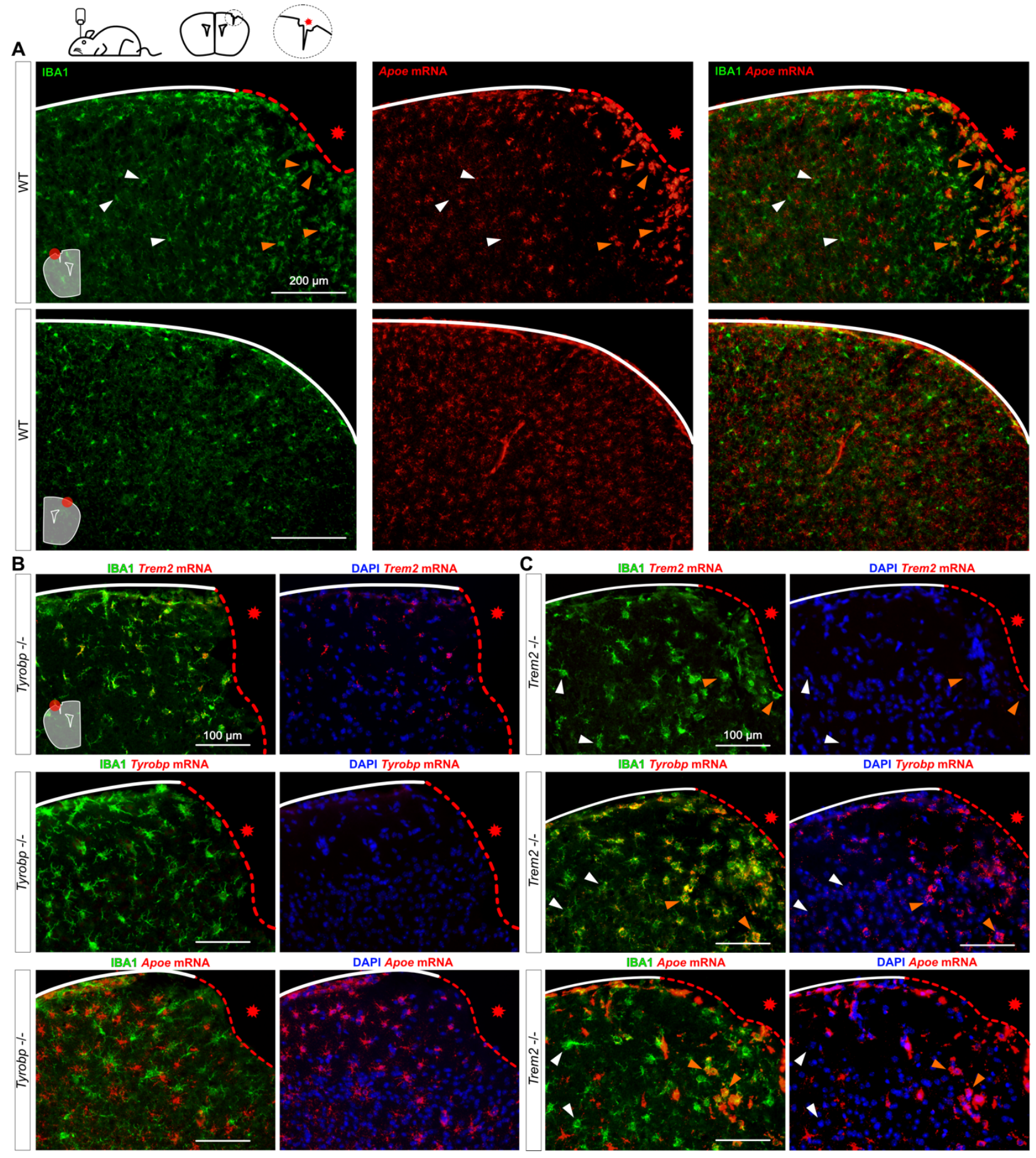

Figure 5: Increases of Tyrobp and Apoe mRNAs in microglia recruited toward a site of stab injury are Trem2-independent

(A) Stab-injured WT mice were sacrificed 3 days after injury and dual RNA fluorescent in situ hybridization and immunohistochemistry for Apoe mRNA (red) and anti-IBA1 (green) respectively was performed. The injured ipsilateral area (red dotted line) is shown on the top row 
and the uninjured contralateral area is show on the bottom row. Scale bar $=200 \mu \mathrm{m}$. (B-C) The same stab injury protocol was utilized in Tyrobp ${ }^{--}$(B) and Trem2 $2^{-/}$(C) mice. Anti-IBA1 and DAPI stainings are shown in green and blue, respectively. Top row: Trem 2 mRNA (red); middle row: Tyrobp mRNA (red); bottom row: Apoe mRNA (red). Mice were 4 months of age and slice thickness $=10 \mu \mathrm{m}$. The red asterisk indicates the injured side. White and orange arrows indicate examples of non-recruited and recruited microglia, respectively. 


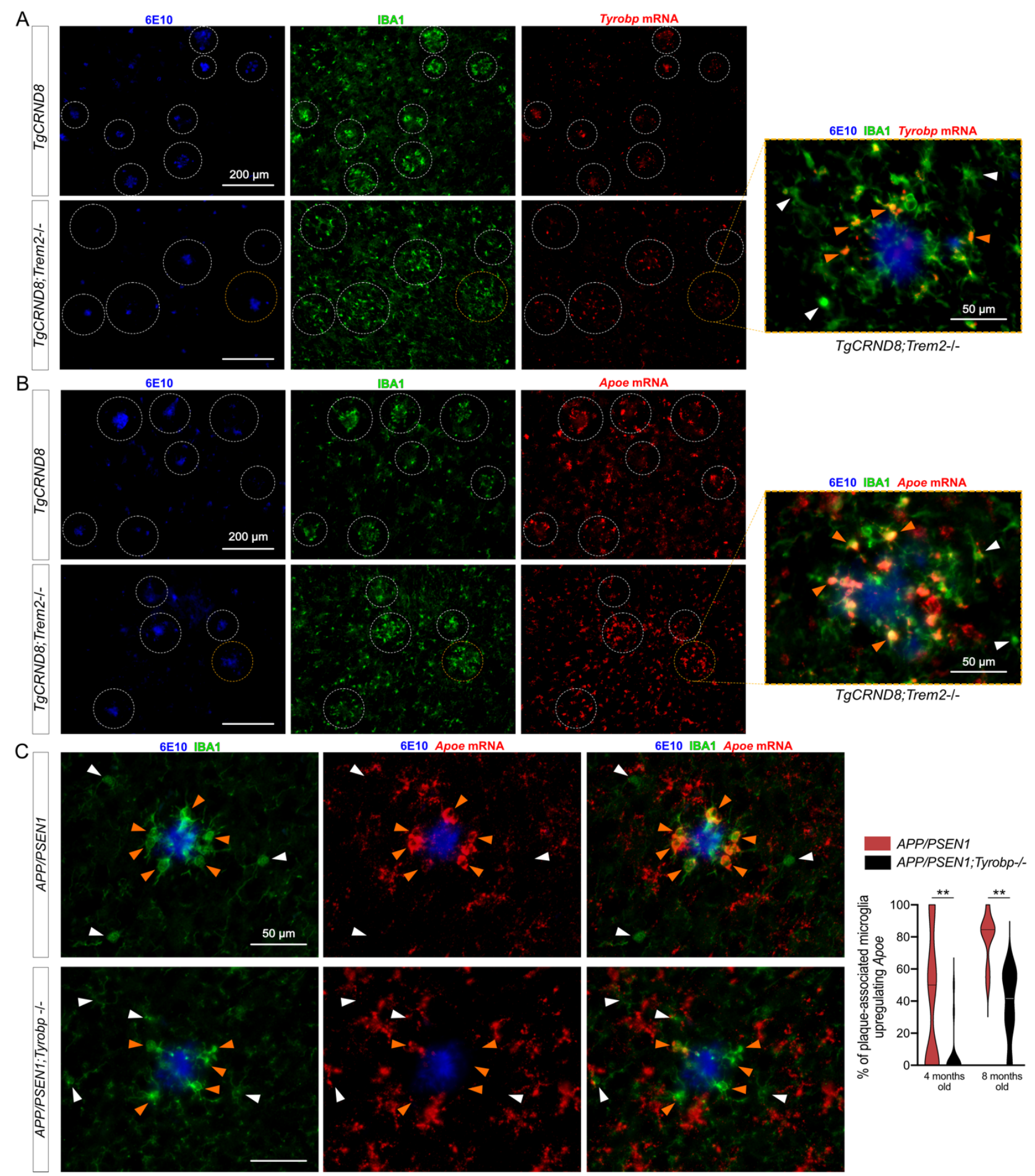

Figure 6: Increases in Tyrobp and Apoe mRNAs in amyloid plaque-associated microglia are

\section{Trem 2 independent}

(A) Dual RNA fluorescent in situ hybridization and immunohistochemistry for Tyrobp mRNA (red), anti-IBA1 (green) and human A $\beta$ (6E10 antibody) (blue) in $\operatorname{TgCRND} 8$ mice on WT (top 
row) or Trem2 $2^{--}$(bottom row) background. Scale bar $=200$ or $50 \mu \mathrm{m}$. (B) Dual RNA fluorescent in situ hybridization and immunohistochemistry for Apoe mRNA (red), anti-IBA1 (green) and human amyloid (6E10 antibody) (blue) in the same mice as in (A). Scale bar $=200$ or $50 \mu \mathrm{m}$. (C) Dual RNA fluorescent in situ hybridization and immunohistochemistry for Apoe mRNA (red), anti-IBA1 (green) and human A $\beta$ (6E10 antibody) (blue) in APP/PSEN1 mice on a WT (top row) or Tyrobp-null (bottom row) background. Scale bar $=50 \mu \mathrm{m}$. Right panel: quantification of the number of plaque-associated microglia with upregulated Apoe mRNA in the same mice as in (D). $\mathrm{N}=2-3$ mice per group (A-C). 


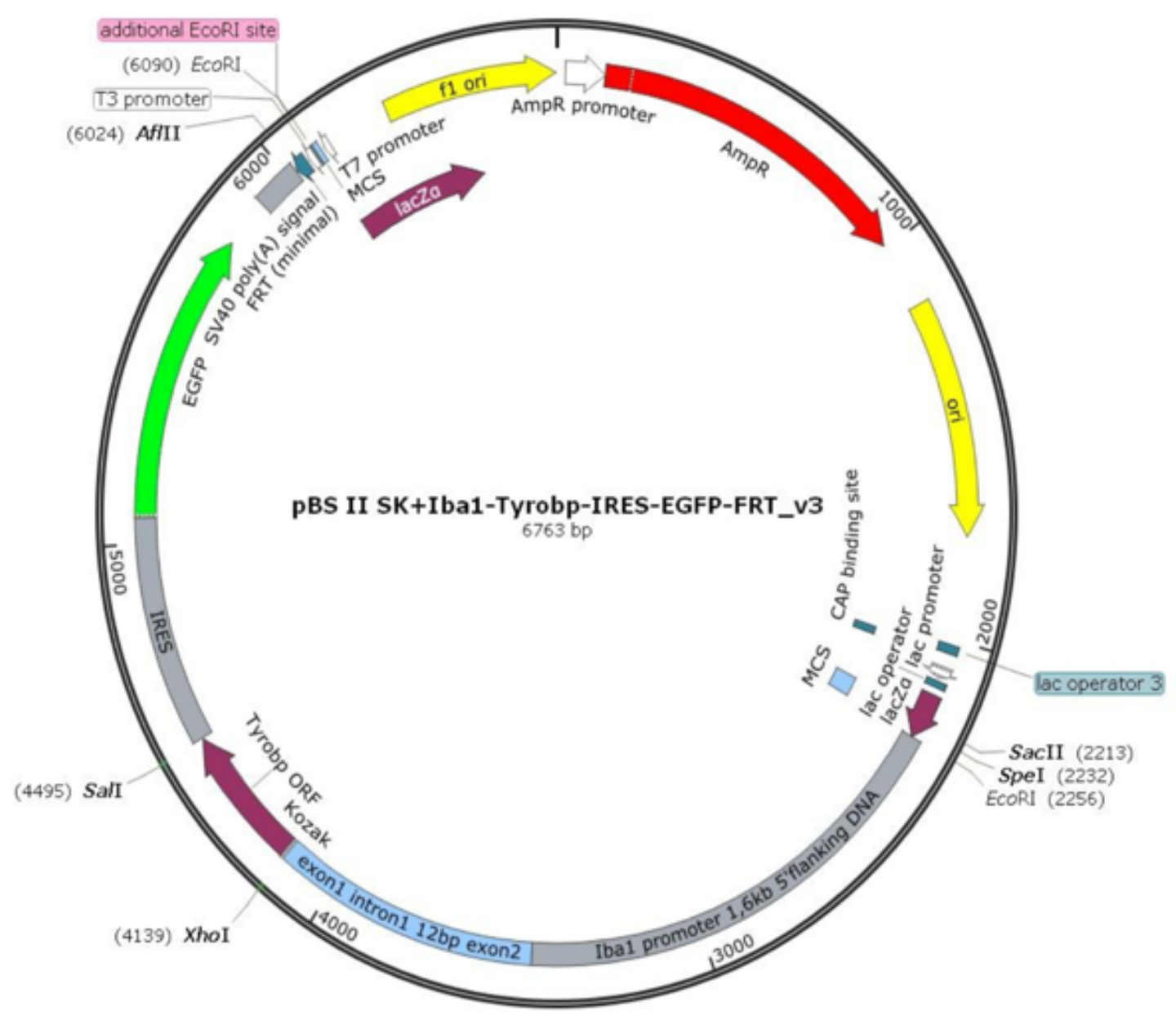

Supplementary Figure 1: Plasmid used to generate the Iba1 ${ }^{\text {Tyrobp }}$ transgenic mice. The insert was linearized after digestion with PacI. 
A
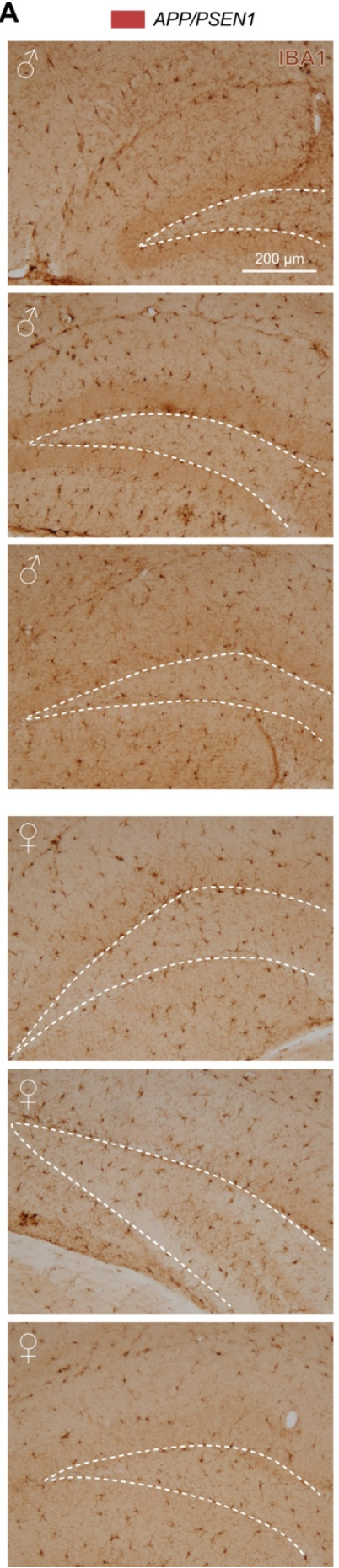

DPAPIPSEN1;Iba1Tyrobp
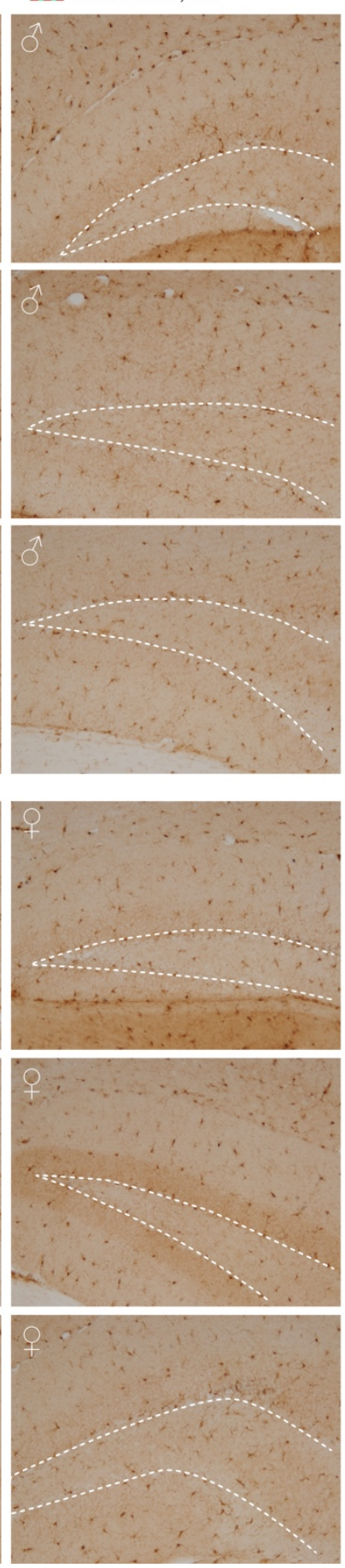

B

MAPT ${ }^{\mathrm{P} 015}$
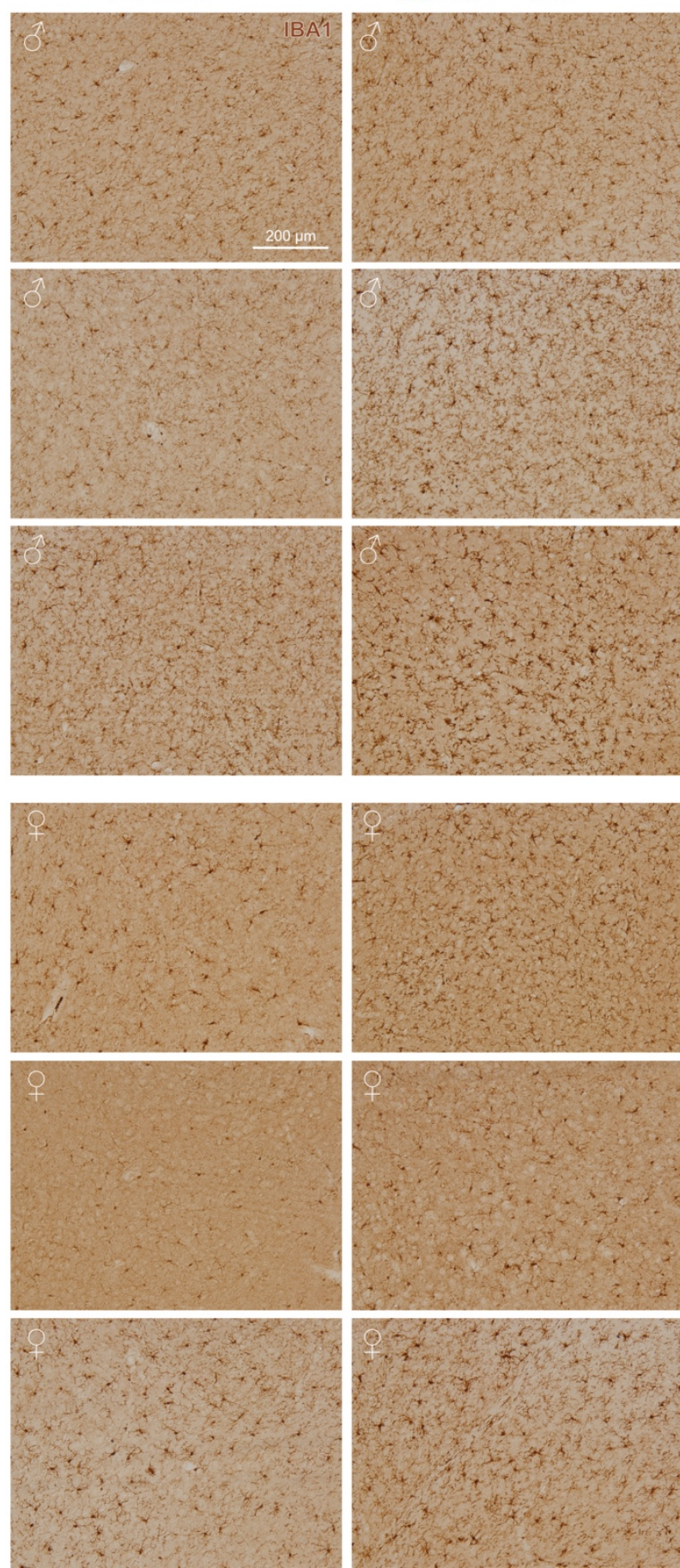

Supplementary Figure 2: Anti-IBA1 immunohistochemistry in 4-month-old APP/PSEN1 and $M A P T^{P 301 S}$ mice with and without the $I b a 1^{T y r o b p}$ transgene

(A) DAB-immunohistochemistry with anti-IBA1 in 4-month-old APP/PSEN1 and APP/PSEN1;Ibal Tyrobp mice. (B) DAB-immunohistochemistry with anti-IBA1 in 4-month-old 
bioRxiv preprint doi: https://doi.org/10.1101/2020.08.18.254649; this version posted August 19,2020. The copyright holder for this preprint

(which was not certified by peer review) is the author/funder, who has granted bioRxiv a license to display the preprint in perpetuity. It is made available under aCC-BY-NC-ND 4.0 International license.

$M A P T^{P 301 S}$ and $M A P T^{P 301 S} ; I b a 1^{\text {Tyrobp }}$ mice. $\mathrm{N}=3$ males and 3 females per genotype. Scale bar $=$ $200 \mu \mathrm{m}$. 
A
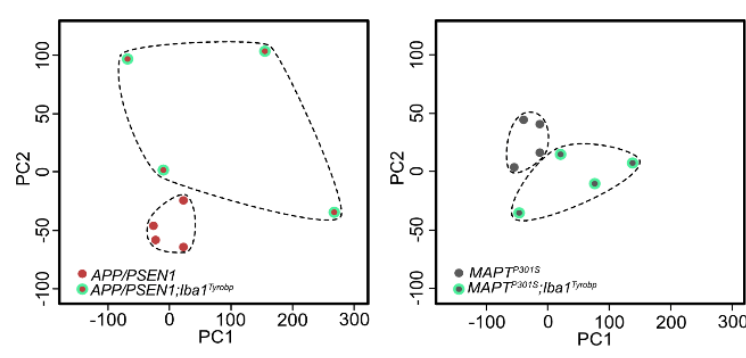

DEGs at $F D R<0.2 \& F C>1.2$

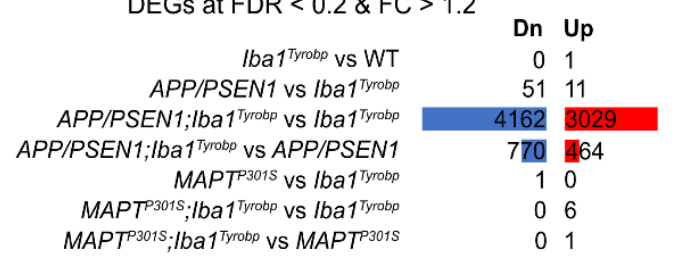

C

\begin{tabular}{ccc}
$\begin{array}{c}\text { Upstream } \\
\text { regulators }\end{array}$ & $\begin{array}{c}\text { Activation } \\
\text { Z-score }\end{array}$ & $\begin{array}{c}\text { p-valye of } \\
\text { overlap }\end{array}$ \\
\hline CREB1 & 3.236 & 0.00000119 \\
SIRT3 & 2.96 & 0.000607 \\
miR-1-3p & 2.527 & 0.00105 \\
CUL4B & 2 & 0.0237 \\
PHF12 & 2 & 0.0586 \\
mir-21 & 2.828 & 0.0866 \\
RPS6KB1 & 2 & 0.125 \\
CREM & 2.333 & 0.154 \\
MAPK1 & 2.304 & 0.175 \\
APOE & 2.44 & 0.224 \\
& & \\
DAP3 & -2.449 & 0.0000104 \\
ALKBH1 & -2 & 0.000243 \\
NSUN3 & -2 & 0.000243 \\
MYC & -2.488 & 0.000244 \\
ADORA2A & -2.021 & 0.00815 \\
JAK1/2 & -2.121 & 0.0139 \\
MIR17HG & -2.53 & 0.0475 \\
NFE2L2 & -2.163 & 0.123 \\
IFNG & -2.488 & 0.143 \\
GAST & -2.236 & 0.297
\end{tabular}

B

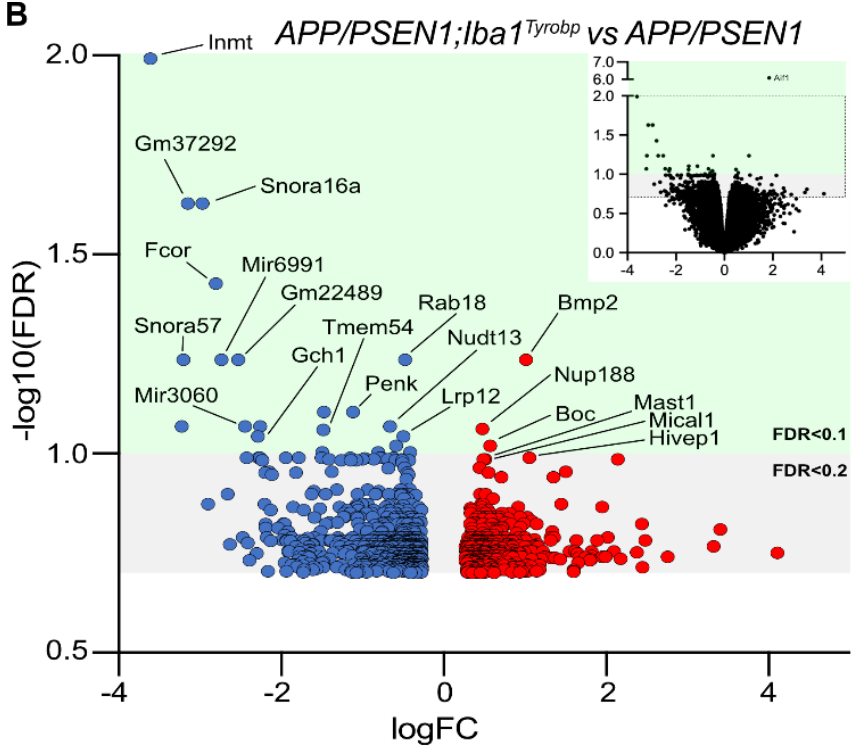

D

\begin{tabular}{lcc}
\multicolumn{1}{c}{ Name } & $\begin{array}{c}\text { p-value } \\
\text { range }\end{array}$ & $\begin{array}{c}\text { \# of } \\
\text { molecules }\end{array}$ \\
\hline Cellular Assembly and Organization & $1.02 \mathrm{E}-02-2.70 \mathrm{E}-09$ & 249 \\
Cellular Function and Maintenance & $9.71 \mathrm{E}-03-2.70 \mathrm{E}-09$ & 195 \\
Cell Mophology & $9.71 \mathrm{E}-03-9.07 \mathrm{E}-09$ & 176 \\
Cellular Development & $9.71 \mathrm{E}-03-9.07 \mathrm{E}-09$ & 171 \\
Cellular Growth and Proliferation & $9.71 \mathrm{E}-03-9.07 \mathrm{E}-09$ & 170
\end{tabular}

Supplementary Figure 3: Bulk RNA sequencing analysis of $A P P / P S E N 1$ or $M A P T^{P 301 S}$ on Ibal ${ }^{\text {Tyrobp }}$ background

(A) RNA sequencing was performed on hippocampi from 4-month-old male WT, Ibal ${ }^{\text {Tyrobp }}$, APP/PSEN1, APP/PSEN1;Ibal ${ }^{\text {Tyrobp }, ~ M A P T ~} T^{P 301 S}$ or MAPT $T^{P 301 S}$;Ibal ${ }^{\text {Tyrobp }}$ mice, $\mathrm{N}=4$ /genotype. Top: Principal Component Analysis (PCA) of the APP/PSEN1 vs APP/PSEN1;Ibal ${ }^{\text {Tyrobp }}$ RNA sequencing samples (left) and $M A P T^{P 301 S}$ vs $M A P T^{P 301 S}$;Ibal ${ }^{\text {Tyrobp }}$ RNA sequencing samples (right). Bottom: Numbers of DEGs (FDR $<0.2$ and FC $>1.2)$ in the genotype comparisons. Blue and red bars represent the number of significantly down- and up-regulated genes, respectively. (B) Volcano plot representation of the DEGs (FDR $<0.2$ and FC $>1.2)$ from the APP/PSEN1; Iba1 $1^{\text {Tyrobp }}$ vs $A P P / P S E N 1$ comparison. The top right quadrant represents the DEGs that have been graphed (all genes with FDR $<0.2$ except Aifl). (C-D) Ingenuity Pathway Analysis (IPA) was used to 
bioRxiv preprint doi: https://doi.org/10.1101/2020.08.18.254649; this version posted August 19, 2020. The copyright holder for this preprint (which was not certified by peer review) is the author/funder, who has granted bioRxiv a license to display the preprint in perpetuity. It is made available under aCC-BY-NC-ND 4.0 International license.

identify the top upstream regulators and predicted activation states $(\mathrm{C})$ as well as the top molecular and cellular functions (D) in the APP/PSEN1;Iba1 ${ }^{\text {Tyrobp }}$ vs APP/PSEN1 comparison. 


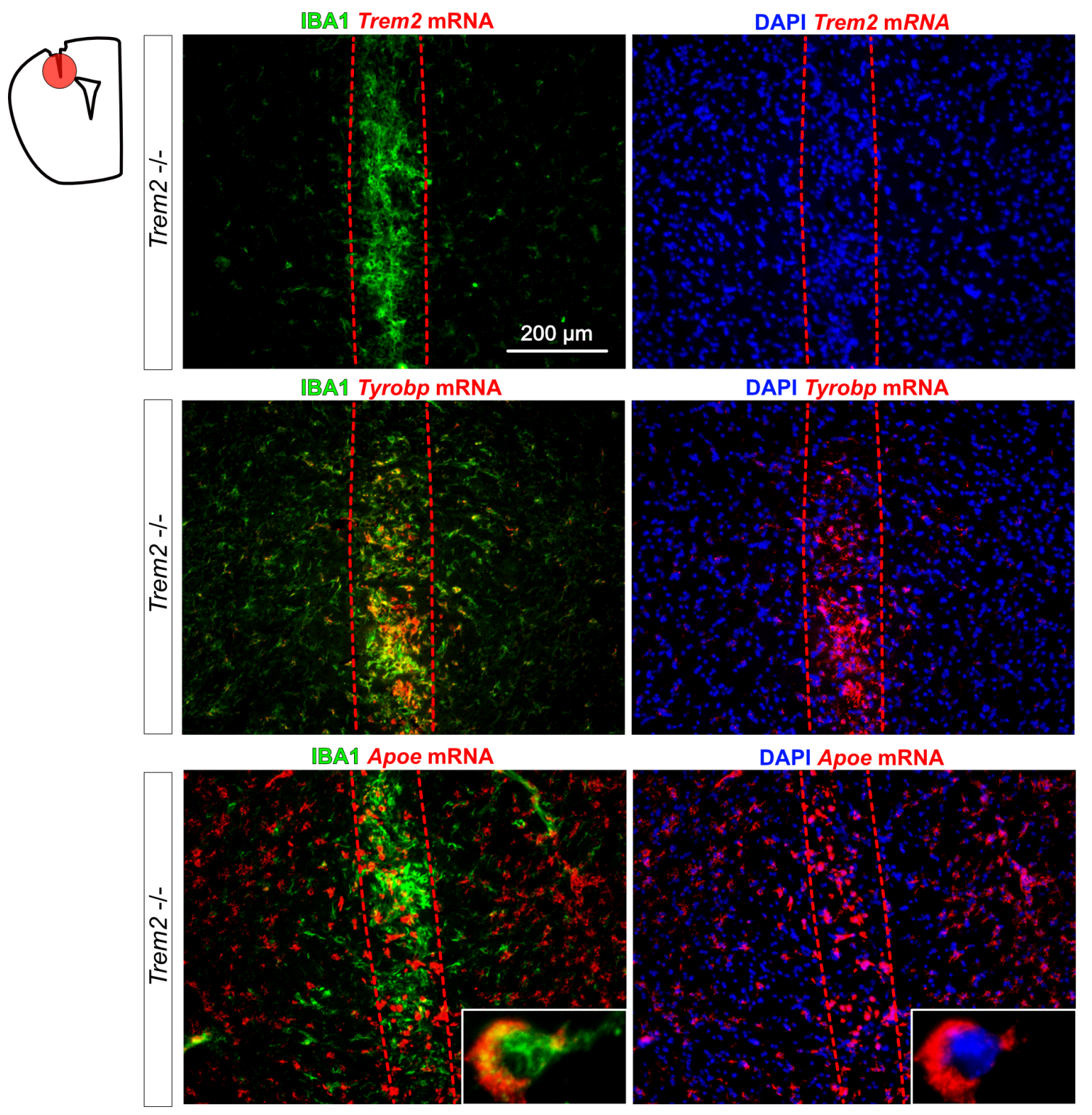

Supplementary Figure 4: Tyrobp and Apoe mRNAs are increased in microglia recruited around the needle tract of stab injured-Trem $2^{-/-}$mice

RNA fluorescent in situ hybridization for Trem2 or Tyrobp or Apoe mRNA double-labeled with an anti-IBA1 antibody (green) or DAPI (blue) in Trem $2^{-/-}$mice injured as described. The red dot line indicates the needle track. Scale bar $=200 \mu \mathrm{m}$. 